\title{
Soft drop
}

\section{Andrew J. Larkoski, ${ }^{a}$ Simone Marzani, ${ }^{b}$ Gregory Soyez ${ }^{c}$ and Jesse Thaler $^{a}$}

\author{
${ }^{a}$ Center for Theoretical Physics, Massachusetts Institute of Technology, \\ Cambridge, MA 02139, U.S.A. \\ ${ }^{b}$ Institute for Particle Physics Phenomenology, Durham University, \\ South Road, Durham DH1 3LE, United Kingdom \\ ${ }^{c}$ IPhT, CEA Saclay, CNRS URA 2306, \\ F-91191 Gif-sur-Yvette, France
}

E-mail: larkoski@mit.edu, simone.marzani@durham.ac.uk, gregory.soyez@cea.fr, jthaler@mit.edu

ABSTRACT: We introduce a new jet substructure technique called "soft drop declustering", which recursively removes soft wide-angle radiation from a jet. The soft drop algorithm depends on two parameters - a soft threshold $z_{\text {cut }}$ and an angular exponent $\beta$ - with the $\beta=0$ limit corresponding roughly to the (modified) mass drop procedure. To gain an analytic understanding of soft drop and highlight the $\beta$ dependence, we perform resummed calculations for three observables on soft-dropped jets: the energy correlation functions, the groomed jet radius, and the energy loss due to soft drop. The $\beta=0$ limit of the energy loss is particularly interesting, since it is not only "Sudakov safe" but also largely insensitive to the value of the strong coupling constant. While our calculations are strictly accurate only to modified leading-logarithmic order, we also include a discussion of higher-order effects such as multiple emissions and (the absence of) non-global logarithms. We compare our analytic results to parton shower simulations and find good agreement, and we also estimate the impact of non-perturbative effects such as hadronization and the underlying event. Finally, we demonstrate how soft drop can be used for tagging boosted $W$ bosons, and we speculate on the potential advantages of using soft drop for pileup mitigation.

KeYwords: QCD Phenomenology, Jets

ARXIV EPRINT: 1402.2657 


\section{Contents}

1 Introduction 2

2 Soft drop declustering $\quad 4$

2.1 Definition 4

2.2 Dependence on $\beta$

$\begin{array}{lll}3 & \text { Energy correlation functions after soft drop } & 7\end{array}$

$\begin{array}{ll}3.1 \text { Leading-order calculation } & 7\end{array}$

3.2 Modified leading logarithmic approximation 9

$\begin{array}{lll}3.3 & \text { Multiple emissions } & 10\end{array}$

$\begin{array}{lll}3.4 & \text { Non-global logarithms } & 12\end{array}$

$\begin{array}{lll}3.5 & \text { Comparison to Monte Carlo } & 14\end{array}$

4 Groomed jet radius $\quad 17$

$\begin{array}{lll}\text { 4.1 Modified leading logarithmic approximation } & 18\end{array}$

$\begin{array}{lll}4.2 & \text { Comparison to Monte Carlo } & 19\end{array}$

5 Jet energy drop $\quad 20$

5.1 Modified leading logarithmic approximation 21

$\begin{array}{ll}5.2 \text { Sudakov safety for } \beta=0 & 22\end{array}$

5.3 Non-global logarithms 23

5.4 Comparison to Monte Carlo 24

6 Non-perturbative contributions $\quad 27$

$\begin{array}{lll}7 & \text { Boosted } W \text { tagging with soft drop } & 27\end{array}$

8 Conclusions $\quad 30$

$\begin{array}{ll}\text { A Details of energy correlation calculation } & 31\end{array}$

B Details of jet radius calculation $\quad 35$

C Details of energy drop calculation $\quad 36$ 


\section{Introduction}

The study of jet substructure has significantly matured over the past five years [1-3], with numerous techniques proposed to tag boosted objects [4-46], distinguish quark from gluon jets [44, 47-51], and mitigate the effects of jet contamination [6, 52-61]. Many of these techniques have found successful applications in jet studies at the Large Hadron Collider (LHC) [50, 62-89], and jet substructure is likely to become even more relevant with the anticipated increase in energy and luminosity for Run II of the LHC.

In addition to these phenomenological and experimental studies of jet substructure, there is a growing catalog of first-principles calculations using perturbative QCD (pQCD). These include more traditional jet mass and jet shape distributions [90-95] as well as more sophisticated substructure techniques [44, 59, 60, 96-103]. Recently, refs. [59, 60] considered the analytic behavior of three of the most commonly used jet tagging/grooming methods - trimming [53], pruning [54, 55], and mass drop tagging [6]. Focusing on groomed jet mass distributions, this study showed how their qualitative and quantitative features could be understood with the help of logarithmic resummation. Armed with this analytic understanding of jet substructure, the authors of ref. [59] developed the modified mass drop tagger (mMDT) which exhibits some surprising features in the resulting groomed jet mass distribution, including the absence of Sudakov double logarithms, the absence of non-global logarithms [104], and a high degree of insensitivity to non-perturbative effects.

In this paper, we introduce a new tagging/grooming method called "soft drop declustering", with the aim of generalizing (and in some sense simplifying) the mMDT procedure. Like any grooming method, soft drop declustering removes wide-angle soft radiation from a jet in order to mitigate the effects of contamination from initial state radiation (ISR), underlying event (UE), and multiple hadron scattering (pileup). Given a jet of radius $R_{0}$ with only two constituents, the soft drop procedure removes the softer constituent unless

$$
\text { Soft Drop Condition: } \frac{\min \left(p_{T 1}, p_{T 2}\right)}{p_{T 1}+p_{T 2}}>z_{\text {cut }}\left(\frac{\Delta R_{12}}{R_{0}}\right)^{\beta}
$$

where $p_{T i}$ are the transverse momenta of the constituents with respect to the beam, $\Delta R_{12}$ is their distance in the rapidity-azimuth plane, $z_{\text {cut }}$ is the soft drop threshold, and $\beta$ is an angular exponent. By construction, eq. (1.1) fails for wide-angle soft radiation. The degree of jet grooming is controlled by $z_{\text {cut }}$ and $\beta$, with $\beta \rightarrow \infty$ returning back an ungroomed jet. As we explain in section 2 , this procedure can be extended to jets with more than two constituents with the help of recursive pairwise declustering. ${ }^{1}$

Following the spirit of ref. [59], the goal of this paper is to understand the analytic behavior of the soft drop procedure, particularly as the angular exponent $\beta$ is varied. There are two different regimes of interest. For $\beta>0$, soft drop declustering removes soft radiation from a jet while still maintaining a fraction (controlled by $\beta$ ) of the soft-collinear radiation. One of the consequences is that the soft drop procedure gives infrared/collinear

\footnotetext{
${ }^{1}$ The soft drop procedure takes some inspiration from the "semi-classical jet algorithm" [58], where a variant of eq. (1.1) with $z_{\text {cut }}=1 / 2$ and $\beta=3 / 2$ is tested at each stage of recursive clustering (unlike declustering considered here).
} 
(IRC) safe results even on a jet with just one constituent. In this regime, soft drop acts like a "groomer", meaning that it changes the constituents of a jet without affecting the overall jet production cross section. For $\beta<0$, soft drop declustering can remove both soft and collinear radiation. For a jet to pass the soft drop procedure, it must have at least two constituents satisfying eq. (1.1). Thus, in this regime, soft drop acts like a "tagger", since it vetoes jets that do not have two well-separated hard prongs. Roughly speaking, the boundary $\beta=0$ corresponds to mMDT, which acts like a tagger at any fixed-order in an $\alpha_{s}$ expansion, but can be thought of as a "Sudakov safe" [105] groomer when all orders in $\alpha_{s}$ are considered.

To demonstrate the behavior of the soft drop procedure, we will present three calculations performed on soft-dropped jets.

- Energy correlation functions. The generalized energy correlation functions (ECF) were introduced in ref. [44], where $\operatorname{ECF}(N, \alpha)$ corresponds to an $N$-point correlation function with angular exponent $\alpha$. In this paper, we will focus on the 2-point correlator through the combination $C_{1}^{(\alpha)} \equiv \operatorname{ECF}(2, \alpha) / \operatorname{ECF}(1, \alpha)^{2}$ (see also refs. [32, 106]). For a jet with two constituents,

$$
C_{1}^{(\alpha)} \simeq \frac{p_{T 1} p_{T 2}}{\left(p_{T 1}+p_{T 2}\right)^{2}}\left(\frac{\Delta R_{12}}{R_{0}}\right)^{\alpha},
$$

where we have added an extra $R_{0}$ normalization factor for later convenience. The value $\alpha=2$ is related to jet thrust/mass [7,91, 107], $\alpha=1$ is related to jet broadening/girth/width [25, 47], and arbitrary $\alpha>0$ is related to the recoil-free angularities [102]. In section 3 , we calculate $C_{1}^{(\alpha)}$ in the modified leading logarithmic (MLL) approximation, which accounts for all terms $\alpha_{s}^{n} L^{2 n-q}$ with $q=0,1$ and $L \equiv \log \left(1 / C_{1}^{(\alpha)}\right)$ in the expansion of the $C_{1}^{(\alpha)}$ cumulative distribution. We will also compute higher-order effects due to multiple emissions and we will find an interesting interplay between the ECF exponent $\alpha$ and the soft drop exponent $\beta$, especially as relates to non-global logarithms.

- Groomed jet radius. The soft drop declustering procedure terminates when eq. (1.1) is satisfied, and the corresponding $\Delta R_{12}$ gives the effective radius $R_{g}$ of the groomed jet. Roughly speaking, the active jet area [108] is $\simeq \pi R_{g}^{2}$. In section 4 , we calculate the $R_{g}$ distribution to MLL accuracy to gain an understanding of how the soft drop procedure might perform in a pileup environment.

- Jet energy drop. Strictly speaking, the groomed jet energy distribution after mMDT (i.e. $\beta=0$ ) is not IRC safe. One of the motivations for introducing the generalized soft drop procedure with $\beta>0$ is to have a method (in the same spirit of trimming [53]) that gives IRC safe distributions for any (otherwise) IRC safe observable measured on groomed jets. In section 5, we calculate the fractional drop in the jet energy after the soft drop procedure to MLL accuracy, including higher-order corrections due to multiple emissions. Intriguingly, we will find that the $\beta \rightarrow 0$ limit is "Sudakov safe" [105], and the resulting jet energy drop spectrum is independent of $\alpha_{s}$ in the fixed coupling approximation. 
While the focus of this paper is on the analytic properties of the soft drop procedure, we will cross check our results using parton shower Monte Carlo simulations. In addition to these analytic studies, we will perform a Monte Carlo study of non-perturbative corrections (hadronization and UE) in section 6, and estimate the tagging performance of soft drop for boosted $W$ bosons in section 7 . We present our conclusions in section 8 .

\section{Soft drop declustering}

\subsection{Definition}

The starting point for soft drop declustering is a jet with characteristic radius $R_{0}$. For definiteness, we will always consider jets defined with the anti- $k_{t}$ algorithm [109], but other jet algorithms would work equally well. We then recluster the jet constituents using the Cambridge-Aachen (C/A) algorithm $[110,111]$ to form a pairwise clustering tree with an angular-ordered structure.

The soft drop declustering procedure depends on two parameters, a soft threshold $z_{\text {cut }}$ and an angular exponent $\beta$, and is implemented as follows:

1. Break the jet $j$ into two subjets by undoing the last stage of $\mathrm{C} / \mathrm{A}$ clustering. Label the resulting two subjets as $j_{1}$ and $j_{2}$.

2. If the subjets pass the soft drop condition $\left(\frac{\min \left(p_{T 1}, p_{T 2}\right)}{p_{T 1}+p_{T 2}}>z_{\text {cut }}\left(\frac{\Delta R_{12}}{R_{0}}\right)^{\beta}\right.$, see eq. (1.1)) then deem $j$ to be the final soft-drop jet. (Optionally, one could also impose the massdrop condition $\max \left(m_{1}, m_{2}\right)<\mu m$ as in ref. [6], but we will not use that here.)

3. Otherwise, redefine $j$ to be equal to subjet with larger $p_{T}$ and iterate the procedure.

4. If $j$ is a singleton and can no longer be declustered, then one can either remove $j$ from consideration ("tagging mode") or leave $j$ as the final soft-drop jet ("grooming mode").

By building a $\mathrm{C} / \mathrm{A}$ tree, we can apply the pairwise soft drop condition from eq. (1.1) to a jet with more than two constituents. Tagging mode is only IRC safe for $\beta \leq 0$ whereas grooming mode is only IRC safe for $\beta>0$. In this paper, we will typically consider $z_{\text {cut }} \simeq 0.1$ but we will explore a wide range of $\beta$ values. $^{2}$

The above algorithm can be thought of as a generalization of the (modified) massdrop tagger (mMDT) [6,59], with $\beta=0$ roughly corresponding to mMDT itself. There are, however, a few important differences. First, soft drop declustering does not require a mass drop condition (or equivalently, the mass drop parameter $\mu$ is set to unity). As shown in ref. [59], the mass drop condition is largely irrelevant for understanding the analytic behavior of mMDT on quark/gluon jets, so we have decided not to include it in the definition here. Second, we note that the $\beta=0$ limit corresponds to a mMDT variant

\footnotetext{
${ }^{2}$ Throughout this paper, we will assume that $\Delta R_{12}<R_{0}$ at every stage of the declustering, such that the algorithm returns the whole jet in the $\beta \rightarrow \infty$ limit. In practice, it is possible for a jet of characteristic radius $R_{0}$ to have $\Delta R_{12}>R_{0}$ when reclustered with $\mathrm{C} / \mathrm{A}$, and in that case we simply apply step 2 without change, such that wide angle emissions can still be vetoed even in the $\beta \rightarrow \infty$ limit.
} 
where step 2 is implemented directly on the transverse momentum fractions of subjets, rather than indirectly through a ratio of a $k_{t}$-distance to a mass [59]. Of course, the two give the same behavior in the small $\min \left(p_{T 1}, p_{T 2}\right) /\left(p_{T 1}+p_{T 2}\right)$ limit, but eq. (1.1) makes it obvious that the soft drop condition drops soft radiation (true to its name). Finally and most importantly, for $\beta \neq 0$, the soft drop condition involves a relation between energies and angular distances, rather than just energies as is the case for $\beta=0$. It is this additional angular dependence (exploited by the exponent $\beta$ ) that we wish to highlight in this paper.

As mentioned in footnote 1 , the soft drop condition takes some inspiration from the "semi-classical jet algorithm" [58]. The semi-classical algorithm is a pairwise clustering algorithm that only allows mergings which satisfy

$$
\text { Semi-classical Condition: } \frac{\min \left(m_{T 1}, m_{T 2}\right)}{m_{T 1}+m_{T 2}}>\frac{1}{2}\left(\frac{\Delta R_{12}}{R_{0}}\right)^{3 / 2},
$$

where $m_{T i}=\sqrt{m_{i}^{2}+p_{T i}^{2}}$. Apart from the change of $p_{T i} \rightarrow m_{T i}$, the semi-classical condition looks like the soft drop condition with $\beta=3 / 2$ and $z_{\text {cut }}=1 / 2$, but there is an important difference. For semi-classical jets, one is recursively clustering a jet using a novel measure. For soft-drop jets, one is taking an existing jet defined with a traditional algorithm and using soft drop declustering to groom away soft wide-angle emissions. Of course, the distinction between clustering and declustering is irrelevant for a jet with only two constituents, but it is very important for our analytic calculations which only apply to declustering of a $\mathrm{C} / \mathrm{A}_{\text {tree. }}{ }^{3}$

\subsection{Dependence on $\beta$}

Before studying the analytic behavior of soft-drop distributions in detail, it is worth making a few general comments about the expected $\beta$ dependence. For simplicity of discussion, we will work with central jets (i.e. rapidity $y=0)$ with small radius $\left(R_{0} \ll 1\right)$. This way, we can freely exchange transverse momentum $p_{T}$ for energy $E$, as well as rapidity-azimuth distance $R$ for opening angle $\theta$. All of the results of this paper extend to non-zero rapidity as well, up to power corrections in the jet radius, which we neglect.

In figure 1, we show the phase space for a single gluon emission from an eikonal hard quark/gluon on the $\left(\log \frac{1}{z}, \log \frac{R_{0}}{\theta}\right)$ plane, where $0 \leq z \leq 1$ is the energy fraction and $0 \leq \theta \leq R_{0}$ is the angle of the emission. We have labeled three modes in the phase space: soft, soft-collinear, and collinear. For this paper, we define the various modes in terms of their $z$ and $\theta$ behavior:

$$
\begin{aligned}
\text { soft modes: } & z \rightarrow 0, \theta=\text { constant, } \\
\text { soft-collinear modes: } & z \rightarrow 0, \theta \rightarrow 0 \\
\text { collinear modes: } & z=\text { constant }, \theta \rightarrow 0 .
\end{aligned}
$$

\footnotetext{
${ }^{3}$ In principle, it is possible to use any of the generalized $k_{t}$ algorithms $[112,113]$ to perform the soft drop declustering. The choice of $\mathrm{C} / \mathrm{A}$ is motivated by the approximate angular ordering of emissions in the parton shower.
} 


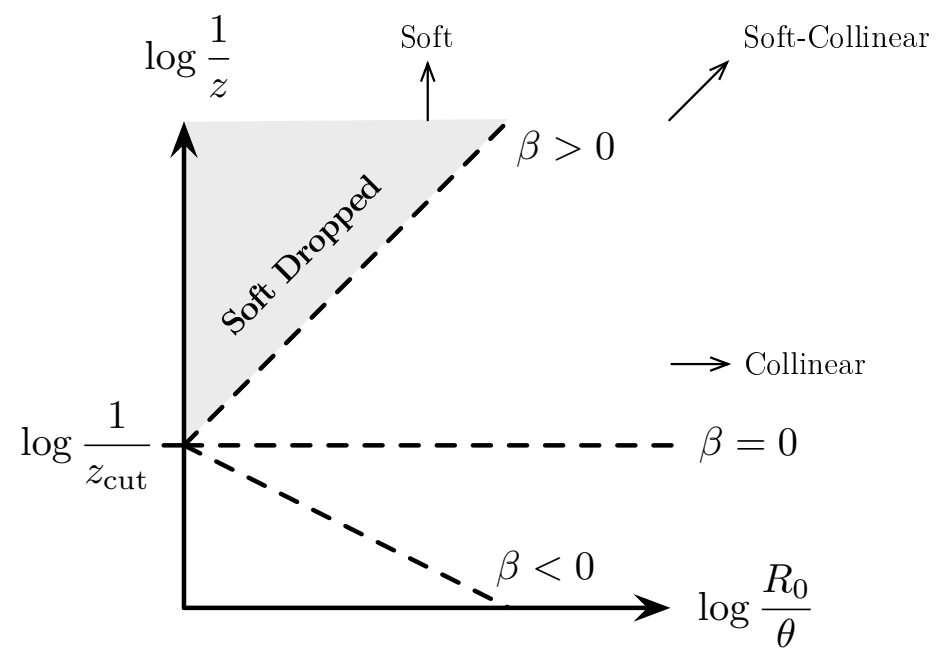

Figure 1: Phase space for emissions on the $\left(\log \frac{1}{z}, \log \frac{R_{0}}{\theta}\right)$ plane. In the strongly-ordered limit, emissions above the dashed line (eq. (2.2)) are vetoed by the soft drop condition. For $\beta>0$, soft emissions are vetoed while much of the soft-collinear region is maintained. For $\beta=0$ (mMDT), both soft and soft-collinear emissions are vetoed. For $\beta<0$, all (two-prong) singularities are regulated by the soft drop procedure.

No relative scaling is assumed between energy fraction $z$ and splitting angle $\theta$ for softcollinear modes. In these logarithmic coordinates, the emission probability is flat in the soft-collinear limit. In the soft limit, the soft drop criteria reduces to

$$
z>z_{\text {cut }}\left(\frac{\theta}{R_{0}}\right)^{\beta} \Rightarrow \log \frac{1}{z}<\log \frac{1}{z_{\text {cut }}}+\beta \log \frac{R_{0}}{\theta} .
$$

Thus, vetoed emissions lie above a straight line of slope $\beta$ on the $\left(\log \frac{1}{z}, \log \frac{R_{0}}{\theta}\right)$ plane, as shown in figure 1.

For $\beta>0$, collinear radiation always satisfies the soft drop condition, so a soft-drop jet still contains all of its collinear radiation. The amount of soft-collinear radiation that satisfies the soft drop condition depends on the relative scaling of the energy fraction $z$ to the angle $\theta$. As $\beta \rightarrow 0$, more of the soft-collinear radiation of the jet is removed, and in the $\beta=0$ (mMDT) limit, all soft-collinear radiation is removed. Therefore, we expect that the coefficient of the double logarithms of observables like groomed jet mass (and $C_{1}^{(\alpha)}$ ) will be proportional to $\beta$, when $\beta$ is small. Similarly, because the soft drop procedure does not change the structure of collinear emissions, observables like the groomed jet energy are IRC safe. Note that running $\beta>0$ soft drop in tagging mode is not IRC safe, since a jet would (would not) be tagged if it contained two (one) collinear particles.

In the strict $\beta=0$ or mMDT limit, collinear radiation is only maintained if $z>z_{\text {cut }}$. Because soft-collinear radiation is vetoed, the resulting jet mass (and $C_{1}^{(\alpha)}$ ) distributions will only exhibit single logarithms, as emphasized in refs. [59, 60]. Because the structure 
of collinear emissions is modified, observables like groomed jet energy are only IRC safe if soft drop is used in tagging mode, since that forces the jet to have a hard two-prong structure, which regulates the collinear singularity. We will see in section 5 , however, that $\beta=0$ grooming mode is still "Sudakov safe" [105].

Finally, for $\beta<0$, there are no logarithmic structures for observables like groomed jet mass at arbitrarily low values of the observable. Effectively, soft drop with negative $\beta$ acts like a cut which enforces $C_{1}^{(\alpha)}>z_{\text {cut }}^{\alpha /|\beta|}$, and this cut regulates the soft-collinear singularities. For example, $\beta=-1$ roughly corresponds to a cut on the relative transverse momentum of the two prongs under scrutiny. Like for $\beta=0, \beta<0$ is only IRC safe in tagging mode.

\section{Energy correlation functions after soft drop}

Generalized energy correlation functions $\operatorname{ECF}(N, \alpha)$ and their double ratios $C_{N-1}^{(\alpha)}$ were introduced in ref. [44] (see also refs. [32, 106] for $N=2$ ). In this paper, we only consider the double ratio for $N=2$ (hereafter referred to as simply the energy correlation functions):

$$
C_{1}^{(\alpha)}=\frac{\operatorname{ECF}(2, \alpha) \operatorname{ECF}(0, \alpha)}{\operatorname{ECF}(1, \alpha)^{2}}
$$

where

$$
\begin{aligned}
& \operatorname{ECF}(0, \alpha)=1, \\
& \operatorname{ECF}(1, \alpha)=\sum_{i \in \text { jet }} p_{T i}, \\
& \operatorname{ECF}(2, \alpha)=\sum_{i<j \in \text { jet }} p_{T i} p_{T j}\left(\frac{\Delta R_{i j}}{R_{0}}\right)^{\alpha} .
\end{aligned}
$$

In this study, we will measure $C_{1}^{(\alpha)}$ on jets which have been groomed according to the soft-drop declustering described above. We will work to lowest non-trivial order in $z_{\text {cut }}$, such that we can ignore the effect of grooming on $\operatorname{ECF}(1, \alpha)$. As stated above, we will focus on central jets $(y=0)$ and assume $R_{0} \ll 1$. In those limits,

$$
C_{1}^{(\alpha)} \simeq \sum_{i<j} z_{i} z_{j}\left(\frac{\theta_{i j}}{R_{0}}\right)^{\alpha},
$$

where $z_{i} \simeq E_{i} / E_{\text {jet }}$ is the energy fraction carried by particle $i$, and $\theta_{i j}$ is the opening angle between particles $i$ and $j$. Up to power-suppressed effects in $R_{0}$, the results of this paper can be extended to non-zero rapidity $(y \neq 0)$ by simply replacing $\theta_{i j}$ with the rapidity-azimuth distance $R_{i j}$ and the energy fraction $z_{i}$ with the momentum fraction $p_{T i} / p_{T \text { jet }}$.

\subsection{Leading-order calculation}

We start our analysis with a relatively simple calculation, by computing the leading order (LO) contribution to the $C_{1}^{(\alpha)}$ distribution in the collinear limit. This limit is appropriate for the small $R_{0}$ assumption considered throughout this paper. 
At LO, the jet consists of only two partons at an angular distance $\Delta R_{12} \simeq \theta$, which carry fractions $z$ and $(1-z)$ of the jet's energy. To have a non-zero contribution to $C_{1}^{(\alpha)}$, both partons must pass the soft-drop condition. In the collinear limit, the groomed $C_{1}^{(\alpha)}$ distribution is

$$
\begin{array}{r}
\frac{1}{\sigma} \frac{d \sigma^{\mathrm{LO}}}{d C_{1}^{(\alpha)}}=\frac{\alpha_{s}}{\pi} \int_{0}^{R_{0}} \frac{d \theta}{\theta} \int_{0}^{1} d z p_{i}(z) \Theta\left(z-z_{\text {cut }}\left(\frac{\theta}{R_{0}}\right)^{\beta}\right) \Theta\left(1-z-z_{\text {cut }}\left(\frac{\theta}{R_{0}}\right)^{\beta}\right) \\
\times \delta\left(C_{1}^{(\alpha)}-z(1-z)\left(\frac{\theta}{R_{0}}\right)^{\alpha}\right),
\end{array}
$$

where $p_{i}(z)$ is the appropriate splitting function for a quark-initiated jet $(i=q)$ or a gluoninitiated jet $(i=g)$, as defined in eq. (A.4). The two theta functions impose the soft drop condition, and the delta function implements the $C_{1}^{(\alpha)}$ measurement.

Because we work in the limit where $C_{1}^{(\alpha)} \ll z_{\text {cut }} \ll 1$, we can ignore terms suppressed by powers of $z_{\text {cut }}$ (but we do not need to resum logarithms of $z_{\text {cut }}$ ); this implies that we can ignore the second theta function in eq. (3.4). Only focusing on the logarithmicallyenhanced contributions, we can also drop the factor of $(1-z)$ in the delta function. These simplifications lead to

$$
\frac{1}{\sigma} \frac{d \sigma^{\mathrm{LO}}}{d C_{1}^{(\alpha)}} \simeq \frac{\alpha_{s}}{\pi} \int_{0}^{R_{0}} \frac{d \theta}{\theta} \int_{0}^{1} d z p_{i}(z) \Theta\left(z-z_{\text {cut }}\left(\frac{\theta}{R_{0}}\right)^{\beta}\right) \delta\left(C_{1}^{(\alpha)}-z\left(\frac{\theta}{R_{0}}\right)^{\alpha}\right) .
$$

For $\beta \geq 0$, the evaluation of the two integrals is straightforward:

$$
\beta \geq 0: \quad \frac{C_{1}^{(\alpha)}}{\sigma} \frac{d \sigma^{\mathrm{LO}}}{d C_{1}^{(\alpha)}} \simeq \frac{\alpha_{s} C_{i}}{\pi} \frac{2}{\alpha} \times \begin{cases}\log \frac{1}{C_{1}^{(\alpha)}}+B_{i}, & C_{1}^{(\alpha)}>z_{\mathrm{cut}} \\ \frac{\beta}{\alpha+\beta} \log \frac{1}{C_{1}^{(\alpha)}}+\frac{\alpha}{\alpha+\beta} \log \frac{1}{z_{\mathrm{cut}}}+B_{i}, & C_{1}^{(\alpha)}<z_{\mathrm{cut}}\end{cases}
$$

up to terms that are power-suppressed in $C_{1}^{(\alpha)}$ or $z_{\text {cut }}$. Here, $C_{i}$ is the overall color factor for the jet $\left(C_{q}=C_{F}=4 / 3\right.$ for quarks and $C_{g}=C_{A}=3$ for gluons) and $B_{i}$ originates from hard-collinear emissions $\left(B_{q}=-3 / 4\right.$ for quarks and $B_{g}=-\frac{11}{12}+\frac{n_{f}}{6 C_{A}}$ for gluons, where $n_{f}$ is the number of active quark flavors). For $\beta<0$, there is an additional restriction which imposes a minimum allowed value for the observable

$$
\beta<0 \text { : Same as eq. (3.6) with additional cut } C_{1}^{(\alpha)}>z_{\text {cut }}^{\alpha /|\beta|} \text {. }
$$

As often happens for grooming and tagging algorithms [59,60], the $C_{1}^{(\alpha)}$ distribution exhibits a transition point at $C_{1}^{(\alpha)}=z_{\text {cut }}$. Unlike trimming and pruning, though, softdrop energy correlation functions do not exhibit further (perturbative) transition points at lower values of the observable. For $C_{1}^{(\alpha)}>z_{\text {cut }}$, soft drop is not active and we recover the ungroomed result. For $C_{1}^{(\alpha)}<z_{\text {cut }}$, soft drop is active and jets that fail the soft drop condition are either removed from consideration (tagging mode) or assigned $C_{1}^{(\alpha)}=0$ (grooming mode). Note that for $\beta>0$, the logarithmic structure of eq. (3.6) is of the same order on both sides of the transition point, so the overall cumulative distribution exhibits Sudakov double logarithms. The effect of the soft drop procedure is to reduce the coefficient of the double logarithm by a factor of $\beta /(\alpha+\beta)$. 


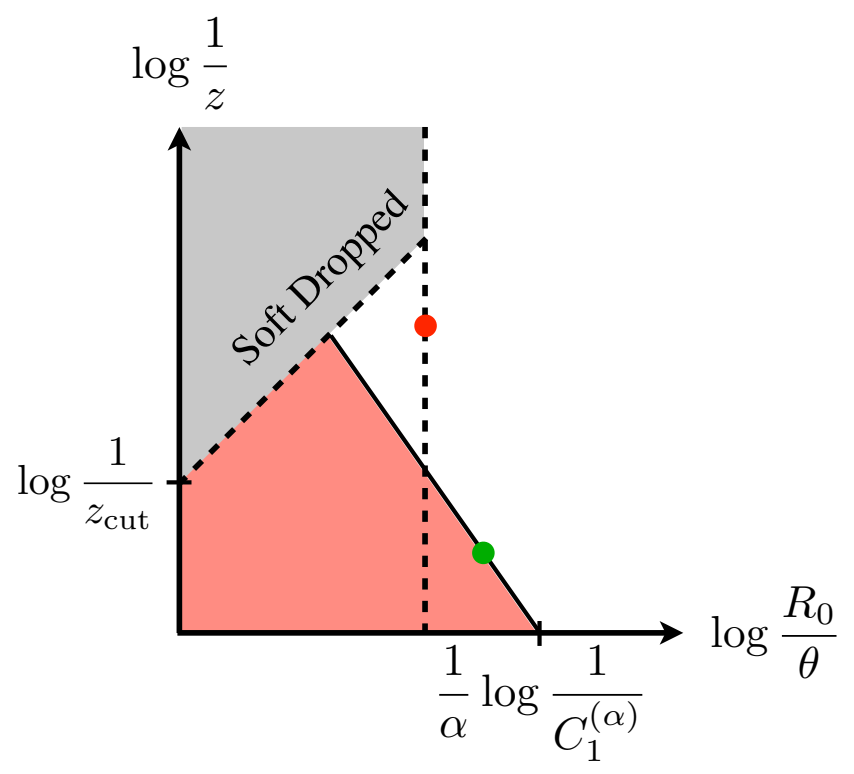

Figure 2: Phase space for emissions relevant for $C_{1}^{(\alpha)}$ in the $\left(\log \frac{1}{z}, \log \frac{R_{0}}{\theta}\right)$ plane. The soft dropped region is gray and the first emission satisfying the soft drop criteria is illustrated by the red dot. The leading emission for $C_{1}^{(\alpha)}$ is illustrated by the green dot with the forbidden emission region (the Sudakov exponent) shaded in pink.

It is instructive to take different limits of the result in eq. (3.6). Consider the $\beta \rightarrow \infty$ limit at fixed $\alpha$ and $z_{\text {cut }}$. This limit should correspond to no grooming, and indeed, in this limit, we recover the expected LO result for the energy correlation function of the ungroomed jet. Now consider the case $\beta=0$, which should correspond to the mMDT limit. This limit kills the logarithmic contribution for $C_{1}^{(\alpha)}<z_{\text {cut }}$, which results in a cumulative distribution that only has single logarithms in $C_{1}^{(\alpha)}$. This result is the generalization to $C_{1}^{(\alpha)}$ of the fact that the mMDT jet mass distribution (here $\alpha=2$ ) is only single logarithmic $[59,60]$.

\subsection{Modified leading logarithmic approximation}

Because of the potentially large logarithms $L \equiv \log \left(1 / C_{1}^{(\alpha)}\right)$ in eq. (3.6), we need to perform some kind of resummation in order to get realistic predictions for the $C_{1}^{(\alpha)}$ distribution. Here, we investigate a simple approximation to the all-order $C_{1}^{(\alpha)}$ distribution by working to modified leading logarithmic (MLL) accuracy, i.e. we aim to capture the terms $\alpha_{s}^{n} L^{2 n-q}$ with $q=0,1$ in the expansion of the cumulative distribution $\Sigma\left(C_{1}^{(\alpha)}\right)$, which gives the probability for the observable to be less than a given value $C_{1}^{(\alpha)}$.

To MLL order, we need to consider the independent emission of any number of soft or collinear gluons within a jet, with the scale of the (one-loop) coupling chosen at the relative transverse momentum scale $\kappa$ of the splitting. In the collinear approximation used throughout this paper, we have $\kappa=z \theta p_{T \text { jet }}$ for jets at arbitrary rapidity.

Virtual emissions are associated with $C_{1}^{(\alpha)}=0$ and therefore always contribute to 
$\Sigma\left(C_{1}^{(\alpha)}\right)$. A real emission contributes to $\Sigma\left(C_{1}^{(\alpha)}\right)$ either if it has been groomed away or if it lies at an angle smaller than the first emission that passes the soft drop condition. This is illustrated in figure 2. Note that the dominant emission contributing to the value of $C_{1}^{(\alpha)}$ must always lie at an angle less than or equal to the angle of the first emission that passes the soft drop condition, so we do not need to consider the correlation of the groomed jet radius and the value of $C_{1}^{(\alpha)}$.

The details of the MLL calculation and explicit results are presented in appendix A. After one explicitly does the sum over all included real emissions, the cumulative distribution can be interpreted in terms of the phase space for vetoed real emissions. This gives the Sudakov exponent

$$
\Sigma\left(C_{1}^{(\alpha)}\right)=\exp \left[-\frac{1}{\alpha} \int_{C_{1}^{(\alpha)}}^{1} \frac{d c}{c} \int_{\max \left(c, z_{\mathrm{cut}} \frac{\alpha}{\alpha+\beta} c^{\frac{\beta}{\alpha+\beta}}\right)}^{1} d z p_{i}(z) \frac{\alpha_{s}(\kappa)}{\pi}\right],
$$

where we have introduced the convenient change of variables $c=z\left(\frac{\theta}{R_{0}}\right)^{\alpha}$. The integral in the exponent corresponds to real emissions that are not removed by the soft drop procedure, but would give a too large contribution to $C_{1}^{(\alpha)}$ (corresponding to the pink shaded region in figure 2). As expected, the $\beta \rightarrow \infty$ limit corresponds to the ungroomed result, and the $\beta=0$ (mMDT) limit matches the jet mass $(\alpha=2)$ distribution in ref. [59].

To better understand the logarithmic structure of the soft-dropped energy correlation functions, it is instructive to perform the integrals in eq. (3.8) in a fixed coupling approximation. For $\beta \geq 0$, neglecting power-suppressed terms, we obtain

$$
\begin{array}{r}
\Sigma\left(C_{1}^{(\alpha)}\right) \stackrel{\text { f.c. }}{=} \exp \left\{-\frac{\alpha_{s} C_{i}}{\pi} \frac{2}{\alpha}\left[\left(\frac{1}{2} \log ^{2} \frac{1}{C_{1}^{(\alpha)}}+B_{i} \log \frac{1}{C_{1}^{(\alpha)}}\right) \Theta\left(1-C_{1}^{(\alpha)}\right) \Theta\left(C_{1}^{(\alpha)}-z_{\text {cut }}\right)\right.\right. \\
+\left(\frac{\beta}{2(\alpha+\beta)} \log ^{2} \frac{1}{C_{1}^{(\alpha)}}+\frac{\alpha}{\alpha+\beta} \log \frac{1}{z_{\text {cut }}} \log \frac{1}{C_{1}^{(\alpha)}}-\frac{\alpha}{2(\alpha+\beta)} \log ^{2} \frac{1}{z_{\text {cut }}}+B_{i} \log \frac{1}{C_{1}^{(\alpha)}}\right) \\
\left.\left.\times \Theta\left(z_{\text {cut }}-C_{1}^{(\alpha)}\right)\right]\right\}, \quad(3.9)
\end{array}
$$

which is the exponential of the cumulative distribution at LO (i.e. the integral of eq. (3.6)). For $\beta<0$, we find an expression analogous to eq. (3.9), but with a lower bound which enforces $C_{1}^{(\alpha)}>z_{\text {cut }}^{\alpha /|\beta|}$, thus regulating the soft-collinear behavior. The limiting values of eq. (3.9) behave as expected. For $\beta \rightarrow \infty$, the regions above and below $z_{\text {cut }}$ give identical results, so they can be combined to return the ungroomed distribution. For $\beta=0$, the coefficient of the double logarithm in the region $C_{1}^{(\alpha)}<z_{\text {cut }}$ vanishes and we obtain the expected mMDT single logarithmic result.

\subsection{Multiple emissions}

Multiple gluon emissions within a jet can affect the value of $C_{1}^{(\alpha)}$. While this effect is strictly speaking beyond MLL accuracy, it is an important component of a full NLL calculation, so it is worth considering how they might affect the $C_{1}^{(\alpha)}$ distribution. For multiple emissions, 
we need to determine what region of phase space can have several emissions that contribute to the measured value of the observable. To logarithmic accuracy, these emissions must give comparable contributions to the final measured value of the observable.

For the case of the energy correlation function $C_{1}^{(\alpha)}$, the region of phase space where multiple emissions contribute can be seen in figure 2. For the green emission that sets the value of $C_{1}^{(\alpha)}$, multiple emissions that contribute logarithmically must lie near the diagonal line defining a fixed value for $C_{1}^{(\alpha)}$. Everywhere along this diagonal line satisfies the soft drop groomer, and therefore all emissions that contribute to the value of $C_{1}^{(\alpha)}$ pass the soft drop phase space requirements. ${ }^{4}$ Also, because C/A clustering enforces angular ordering, these multiple emissions must lie at angles smaller than the first emission that passes the soft drop requirement. Therefore, accounting for multiple emissions requires including an arbitrary number of emissions that contribute to $C_{1}^{(\alpha)}$ and pass the soft drop requirement.

To single logarithmic accuracy, the cumulative distribution of soft drop groomed $C_{1}^{(\alpha)}$ can then be expressed as an explicit sum over uncorrelated emissions as

$$
\begin{aligned}
\Sigma\left(C_{1}^{(\alpha)}\right)=\sum_{n=1}^{\infty} & \prod_{m=1}^{n}\left[\int_{0}^{R_{0}} \frac{d \theta_{m}}{\theta_{m}} \int_{0}^{1} d z_{m} p_{i}\left(z_{m}\right) \frac{\alpha_{s}\left(\kappa_{m}\right)}{\pi} \Theta\left(z_{m}-z_{\text {cut }}\left(\frac{\theta_{m}}{R_{0}}\right)^{\beta}\right) \Theta\left(\theta_{i-1}-\theta_{i}\right)\right] \\
& \times \Theta\left(C_{1}^{(\alpha)}-\sum_{m=1}^{n} z_{m}\left(\frac{\theta_{m}}{R_{0}}\right)^{\alpha}\right) e^{-\int_{0}^{R_{0}} \frac{d \theta}{\theta} \int_{0}^{1} d z p_{i}(z) \frac{\alpha_{s}(\kappa)}{\pi} \Theta\left(z-z_{\text {cut }}\left(\frac{\theta}{R_{0}}\right)^{\beta}\right)} .
\end{aligned}
$$

The requirement $\Theta\left(\theta_{i-1}-\theta_{i}\right)$ imposes angular ordering and the explicit exponential is the sum of virtual contributions. The explicit sum can be evaluated by a Laplace transformation which yields

$$
\Sigma\left(C_{1}^{(\alpha)}\right)=\int \frac{d \nu}{2 \pi i \nu} e^{\nu C_{1}^{(\alpha)}} e^{-R\left(\nu^{-1}\right)}
$$

where the $\nu$ integral represents the inverse Laplace transform. The function $R\left(\nu^{-1}\right)$ is called the radiator and is

$$
R\left(\nu^{-1}\right)=\int_{0}^{R_{0}} \frac{d \theta}{\theta} \int_{0}^{1} d z p(z) \frac{\alpha_{s}(\kappa)}{\pi} \Theta\left(z-z_{\text {cut }}\left(\frac{\theta}{R_{0}}\right)^{\beta}\right)\left(1-\exp \left[-\nu z\left(\frac{\theta}{R_{0}}\right)^{\alpha}\right]\right) .
$$

Because they are Laplace conjugates of one another, logarithmic accuracy in $C_{1}^{(\alpha)}$ corresponds to the same logarithmic accuracy in $\nu$. Therefore, for single logarithmic accuracy in $C_{1}^{(\alpha)}$, we must compute the radiator to single logarithmic accuracy in $\nu$. Expanding around $\nu^{-1}=C_{1}^{(\alpha)}$, the inverse Laplace transform can be evaluated explicitly (see e.g. ref. [114116]) and we find

$$
\Sigma\left(C_{1}^{(\alpha)}\right)=\frac{e^{-\gamma_{E} R^{\prime}\left(C_{1}^{(\alpha)}\right)}}{\Gamma\left(1+R^{\prime}\left(C_{1}^{(\alpha)}\right)\right)} e^{-R\left(C_{1}^{(\alpha)}\right)},
$$

\footnotetext{
${ }^{4}$ One might worry that if the emission that sets the value of $C_{1}^{(\alpha)}$ lies near the boundary between the soft-drop groomed region and soft-drop kept region, then emissions that contribute to the observable may not satisfy the soft-drop requirement on their own. While this is true, the contributions from such emissions are subleading to the accuracy to which we work and can therefore be ignored.
} 
where

$$
R\left(C_{1}^{(\alpha)}\right)=\int_{0}^{R_{0}} \frac{d \theta}{\theta} \int_{0}^{1} d z p(z) \frac{\alpha_{s}(\kappa)}{\pi} \Theta\left(z-z_{\text {cut }}\left(\frac{\theta}{R_{0}}\right)^{\beta}\right) \Theta\left(z\left(\frac{\theta}{R_{0}}\right)^{\alpha}-C_{1}^{(\alpha)}\right)
$$

$\gamma_{E}$ is the Euler-Mascheroni constant, $\Gamma$ is the gamma function, and

$$
R^{\prime}\left(C_{1}^{(\alpha)}\right)=-\frac{\partial}{\partial \log C_{1}^{(\alpha)}} R\left(C_{1}^{(\alpha)}\right)
$$

The prefactor in eq. (3.13) containing $R^{\prime}\left(C_{1}^{(\alpha)}\right)$ captures the effect of multiple emissions on the distribution of $C_{1}^{(\alpha)}$. We remind the reader that to single-logarithmic accuracy, we can neglect the hard-collinear contribution in the multiple-emission prefactor, i.e. we can take $B_{i}=0$ in eq. (3.15).

Multiple-emission contributions to the ungroomed $C_{1}^{(\alpha)}$ distribution were considered in ref. [44]. The effect is non-negligible for the jet-mass like case $(\alpha=2)$ and increases as $\alpha$ grows smaller. However, we expect these kind of contributions to be reduced by the soft-drop procedure, essentially because the coefficient of the soft-collinear terms, which give the single-logarithmic contribution to $R^{\prime}$, is reduced by a factor $\mathcal{O}(\beta)$. We shall come back to this discussion in section 3.5, when we compare the resummed calculation to a result obtained with a parton shower event generator.

The differential distribution for the observable $C_{1}^{(\alpha)}$ with multiple emissions, i.e. the derivative of eq. (3.13), depends on the second derivative of the radiator function $R$. However, within our approximations, $R^{\prime \prime}$ is not continuous across $C_{1}^{(\alpha)}=z_{\text {cut }}$ (see for instance eq. (3.9)). Physically, this is a consequence of the fact that emissions that contribute similarly to the observable can occur on either side of the $z_{\text {cut }}$ transition point. As a result, the distribution with multiple emission exhibits a discontinuity at $C_{1}^{(\alpha)}=z_{\text {cut }}$ because of terms which are beyond NLL accuracy in $\log \Sigma$. In order to restore continuity, we can simply replace the logarithmic derivative with its discrete version:

$$
R^{\prime}\left(C_{1}^{(\alpha)}\right) \rightarrow \frac{R\left(C_{1}^{(\alpha)} e^{-\delta}\right)-R\left(C_{1}^{(\alpha)}\right)}{\delta}
$$

The specific choice of $\delta$ is irrelevant to single logarithmic accuracy, and we take $\delta=1$ for definiteness. One can think of the $\delta$-dependence as being one source of theoretical uncertainty.

\subsection{Non-global logarithms}

The jet-based $C_{1}^{(\alpha)}$ is an example of a non-global observable [104], meaning that it receives single-logarithmic contributions coming from an ensemble of gluons that are outside of the jet which then radiate soft gluons into the jet. The resummation of non-global logarithms for the specific case of the mass of anti- $k_{t}$ jets $(\alpha=2)$ was performed in refs. [92, 93] in the large $N_{C}$ limit (for recent work at finite $N_{C}$ see ref. [117]). A key result of refs. [59, 60] is that the mass distribution of an mMDT jet is free of non-global logarithms, since the mMDT eliminates all sensitivity to soft emissions. Since non-global logarithms contribute 
only at the single-logarithmic level, they are formally beyond MLL accuracy. That said, it is interesting to study the structure of non-global logarithms for soft-dropped $C_{1}^{(\alpha)}$ as $\beta$ is varied, especially since we know non-global logarithms must vanish at $\beta=0$.

Consider the lowest-order configuration that can produce a non-global logarithm, namely the correlated emission of two gluons where $k_{1}$ is outside the original anti- $k_{t}$ jet and a softer gluon $k_{2}$ is inside it. ${ }^{5}$ To contribute to a non-global logarithm, $k_{2}$ has to pass the soft-drop condition, so the relevant phase space constraints are

$$
\Theta^{\mathrm{NG}} \equiv \Theta\left(z_{1}-z_{2}\right) \Theta\left(\theta_{1}-R_{0}\right) \Theta\left(R_{0}-\theta_{2}\right) \Theta\left(z_{2}-z_{\text {cut }}\left(\frac{\theta_{2}}{R_{0}}\right)^{\beta}\right) .
$$

To extract the non-global contribution, we have consider the $C_{F} C_{A}$ correlated emission term of the squared matrix element for two gluon emissions that satisfy the $\Theta^{\mathrm{NG}}$ constraint:

$$
\frac{1}{\sigma} \frac{d \sigma^{\mathrm{NG}}}{d C_{1}^{(\alpha)}}=4 C_{F} C_{A}\left(\frac{\alpha_{s}}{2 \pi}\right)^{2} \int \frac{d z_{1}}{z_{1}} \frac{d z_{2}}{z_{2}} \int \theta_{2} d \theta_{2} \int \theta_{1} d \theta_{1} \Omega_{2} \Theta^{\mathrm{NG}} \delta\left(C_{1}^{(\alpha)}-z_{2}\left(\frac{\theta_{2}}{R_{0}}\right)^{\alpha}\right)
$$

where $\Omega_{2}$ is the (azimuthally averaged) angular function (see for example [119])

$$
\Omega_{2}=\frac{2}{\left(1-\cos \theta_{1}\right)\left(1+\cos \theta_{2}\right)\left|\cos \theta_{1}-\cos \theta_{2}\right|} \simeq \frac{4}{\theta_{1}^{2}\left(\theta_{1}^{2}-\theta_{2}^{2}\right)} .
$$

It is now relatively easy to evaluate eq. (3.18). For definiteness, we consider $\beta \geq 0$ and obtain

$$
\begin{aligned}
\frac{C_{1}^{(\alpha)}}{\sigma} \frac{d \sigma^{\mathrm{NG}}}{d C_{1}^{(\alpha)}}= & 4 C_{F} C_{A}\left(\frac{\alpha_{s}}{2 \pi}\right)^{2} \int_{R_{0}^{2}}^{1} d \theta_{1}^{2} \int_{0}^{R_{0}^{2}} d \theta_{2}^{2} \Theta\left(\theta_{2}^{\alpha}-R_{0}^{\alpha} C_{1}^{(\alpha)}\right) \Theta\left(R_{0}\left(\frac{C_{1}^{(\alpha)}}{z_{\text {cut }}}\right)^{\frac{1}{\alpha+\beta}}-\theta_{2}\right) \\
& \times \frac{1}{\theta_{1}^{2}\left(\theta_{1}^{2}-\theta_{2}^{2}\right)} \log \frac{\theta_{2}^{\alpha}}{R_{0}^{\alpha} C_{1}^{(\alpha)}} \\
=4 C_{F} C_{A}\left(\frac{\alpha_{s}}{2 \pi}\right)^{2} & {\left[\operatorname{Li}_{2}\left(\left(\frac{C_{1}^{(\alpha)}}{z_{\text {cut }}}\right)^{\frac{2}{\alpha+\beta}}\right) \frac{\alpha \log \frac{1}{z_{\text {cut }}}+\beta \log \frac{1}{C_{1}^{(\alpha)}}}{\alpha+\beta}\right.} \\
+ & \left.\frac{\alpha}{2} \operatorname{Li}_{3}\left(C_{1}^{(\alpha) \frac{2}{\alpha}}\right)-\frac{\alpha}{2} \operatorname{Li}_{3}\left(\left(\frac{C_{1}^{(\alpha)}}{z_{\text {cut }}}\right)^{\frac{2}{\alpha+\beta}}\right)\right]+\mathcal{O}\left(R_{0}^{2}\right) .
\end{aligned}
$$

By itself, eq. (3.20) is not particularly enlightening, so it is instructive to take the no grooming limit $(\beta \rightarrow \infty)$ and the mMDT limit $(\beta=0)$. To get a sensible result, we first take the limit of eq. (3.20) with respect to $\beta$ and then consider the behavior of the resulting expression at small $C_{1}^{(\alpha)}$. For $\beta \rightarrow \infty$,

$$
\lim _{\beta \rightarrow \infty} \frac{C_{1}^{(\alpha)}}{\sigma} \frac{d \sigma^{\mathrm{NG}}}{d C_{1}^{(\alpha)}}=C_{F} C_{A}\left(\frac{\alpha_{s}}{2 \pi}\right)^{2}\left(\frac{2}{3} \pi^{2} \log \frac{1}{C_{1}^{(\alpha)}}+\cdots\right)+\mathcal{O}\left(\beta^{-1}\right),
$$

\footnotetext{
${ }^{5}$ Because the original jet is defined with the anti- $k_{t}$ algorithm, we are not sensitive to clustering logarithms first described in ref. [118].
} 
where the dots indicate terms that are not logarithmically enhanced at small $C_{1}^{(\alpha)}$. Eq. (3.21) is precisely the result for anti- $k_{t}$ jets in the small jet radius limit [92], and extends to all $\alpha>0$ since the non-global logarithms arise from soft wide-angle emissions for which the specific angular exponent is a power correction.

For $\beta=0$, there are no non-global logarithms. In particular, the $\log C_{1}^{(\alpha)}$ term in eq. (3.20) has null coefficient and, after taking the small $C_{1}^{(\alpha)}$ limit, we obtain

$$
\lim _{\beta \rightarrow 0} \frac{C_{1}^{(\alpha)}}{\sigma} \frac{d \sigma^{\mathrm{NG}}}{d C_{1}^{(\alpha)}}=C_{F} C_{A}\left(\frac{\alpha_{s}}{2 \pi}\right)^{2}\left(\frac{C_{1}^{(\alpha)}}{z_{\text {cut }}}\right)^{\frac{2}{\alpha}}\left(4 \log \frac{1}{z_{\text {cut }}}-2 \alpha\left(1-z_{\text {cut }}^{\frac{2}{\alpha}}\right)+\cdots\right)+\mathcal{O}(\beta) .
$$

This expression is consistent with the small- $z_{\text {cut }}$ and small- $R_{0}$ limit of result for the mMDT mass distribution $(\alpha=2)$ [60].

In general, for finite values of $\beta>0$, the non-global logarithms are suppressed by powers of $C_{1}^{(\alpha)}$ with respect to the anti- $k_{t}(\beta \rightarrow \infty)$ case. Taking the small $C_{1}^{(\alpha)}$ limit of eq. (3.20), we find ${ }^{6}$

$$
\lim _{C_{1}^{(\alpha)} \rightarrow 0} \frac{C_{1}^{(\alpha)}}{\sigma} \frac{d \sigma^{\mathrm{NG}}}{d C_{1}^{(\alpha)}}=4 C_{F} C_{A}\left(\frac{\alpha_{s}}{2 \pi}\right)^{2} \frac{\beta}{\alpha+\beta}\left(\frac{C_{1}^{(\alpha)}}{z_{\text {cut }}}\right)^{\frac{2}{\alpha+\beta}} \log \frac{1}{C_{1}^{(\alpha)}}+\mathcal{O}\left(C_{1}^{(\alpha) \frac{2}{\alpha+\beta}}\right) .
$$

Because the non-global logarithms are formally power suppressed, we can consistently neglect their resummation to NLL accuracy. As expected, soft drop declustering removes soft divergences, and hence removes non-global logarithms.

\subsection{Comparison to Monte Carlo}

We conclude our discussion of $C_{1}^{(\alpha)}$ by comparing our analytic MLL calculation in section 3.2 (plus the multiple-emission corrections from section 3.3) to a standard Monte Carlo parton shower. For these simulations, we use PyтнiA 8.175 [120] ( $p_{t}$-ordered shower) with the default $4 \mathrm{C}$ tune [121]. We consider proton-proton collisions at $14 \mathrm{TeV}$ at parton level, including initial- and final-state showering but without multiple parton interactions (i.e. $\mathrm{UE})$. We discuss UE and hadronization corrections in section 6 .

Jets clustering is performed with the anti- $k_{t}$ algorithm [109] with radius $R_{0}=1.0^{7}$ using a development version of FASTJET 3.1 (which for the features used here behaves identically to the 3.0.x series [123]). A transverse momentum selection cut $p_{T}>3 \mathrm{TeV}$ is applied on the jets before grooming. To implement the soft drop procedure described in section 2 , jets are reclustered using exclusive C/A $[110,111]$ to return the same jet. The soft drop code will be made available as part of the FASTJET contrib project (http: //fastjet.hepforge.org/contrib/).

We start by considering the case $\alpha=2$, which corresponds to the familiar case of the jet mass distribution. In figure 3 we show results for $q q \rightarrow q q$ scattering for different values

\footnotetext{
${ }^{6}$ Note that the limits $\beta \rightarrow \infty$ and $C_{1}^{(\alpha)} \rightarrow 0$ do not commute with one another as eq. (3.21) does not follow from the $\beta \rightarrow \infty$ limit of eq. (3.23).

${ }^{7}$ We choose $R_{0}=1.0$ primarily to ease the comparison with previous studies of mMDT in ref. [59]. While we take the small jet radius approximation in this paper, it is known to be reasonable even up to $R_{0} \sim 1$ [93, 122].
} 


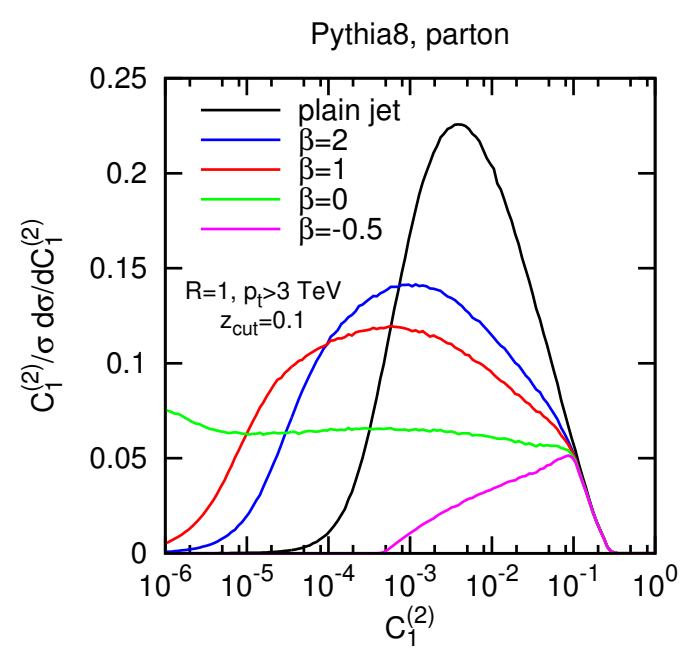

(a)

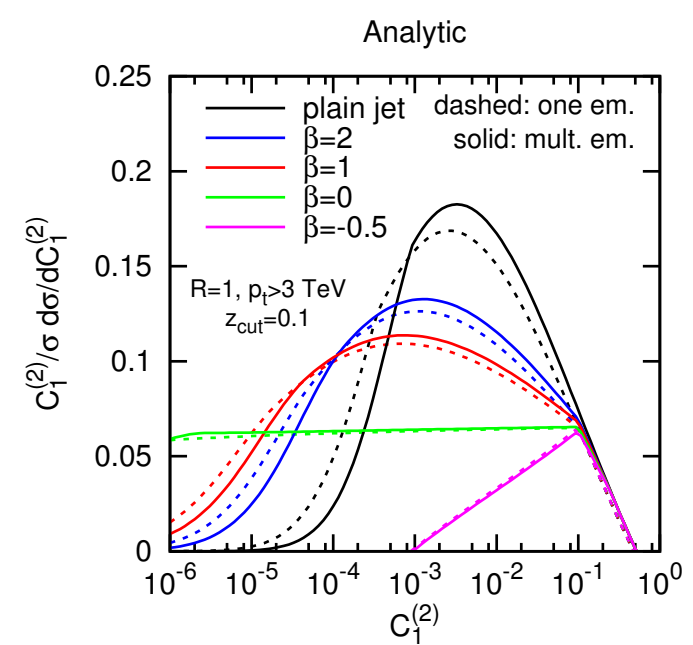

(b)

Figure 3: The energy correlation functions $C_{1}^{(\alpha=2)}$ for quark-initiated jets. Here we compare Pyтнia 8 [120] (left), our MLL formula in eq. (3.8) (right, dashed curves), and our MLL plus multiple-emissions formula in eq. (3.13) (right, solid curves). These $\alpha=2$ curves correspond to the case of jet mass-squared (normalized to jet energy squared). We show both the ungroomed (plain jet) distribution, as well as groomed distributions from soft drop declustering with $z_{\text {cut }}=0.1$ and various values of $\beta$. For $\beta=2,1$, we see the expected Sudakov double logarithmic peaks, while $\beta=0$ (mMDT) has only single logarithms and $\beta=-1$ cuts off at small values. The PyтніA 8 distributions do not have hadronization effects, and the MLL distributions are evaluated by freezing $\alpha_{s}$ in the infrared.

of angular power $\beta$ in the soft-drop declustering procedure $(\beta=0$ is the mMDT already studied in ref. [59]). The plot on the left has been obtained from PyтнiA 8, while the one on the right has been obtained with the analytic resummation, evaluated numerically by freezing the strong coupling in the infrared (see appendix A). Dashed curves correspond to MLL accuracy eq. (3.8), while solid ones include the multiple-emission effect from eq. (3.13).

The plain jet mass case $(\beta \rightarrow \infty$, shown in black) exhibits the characteristic Sudakov peak. All the other curves exhibit a transition point at $C_{1}^{(\alpha)}=z_{\text {cut }}$ and soft drop is active for $C_{1}^{(\alpha)}<z_{\text {cut }}$. Soft-dropped distributions with $\beta>0$ (blue and red) are double logarithmic and indeed we can recognize this behavior in the shape of the distribution (i.e. an upside-down parabola on a log-linear plot). The case $\beta=0$ (mMDT, green) has no soft logarithms, so the resulting distribution is single logarithmic. The distribution is nearly flat because the choice $z_{\text {cut }}=0.1$ is close to the value that minimizes higher-order corrections for quark-initiated jets [59]. As discussed in eq. (3.7), the choice of negative $\beta$ (here $\beta=-0.5$ in pink) leads to a distribution with a minimum allowed value, thus regulating both soft and collinear divergencies.

For the groomed distributions, there is good agreement between the parton shower 


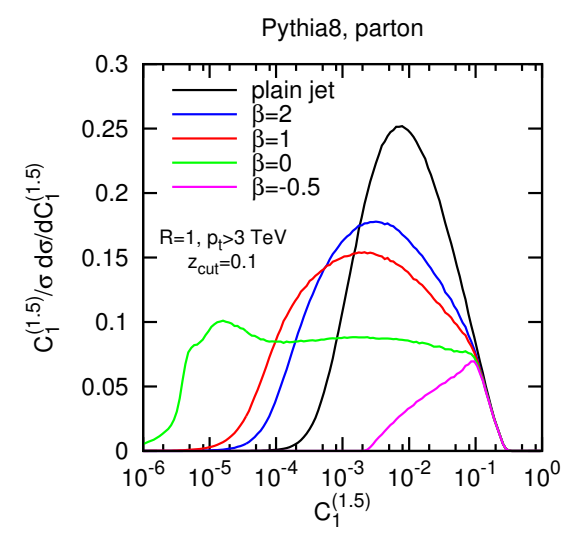

(a)

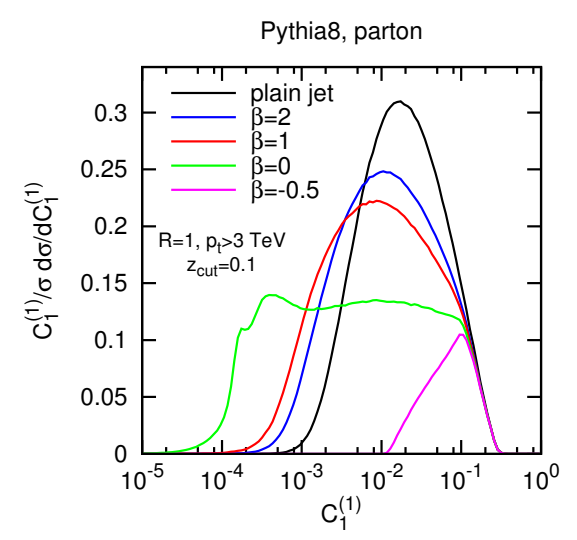

(c)

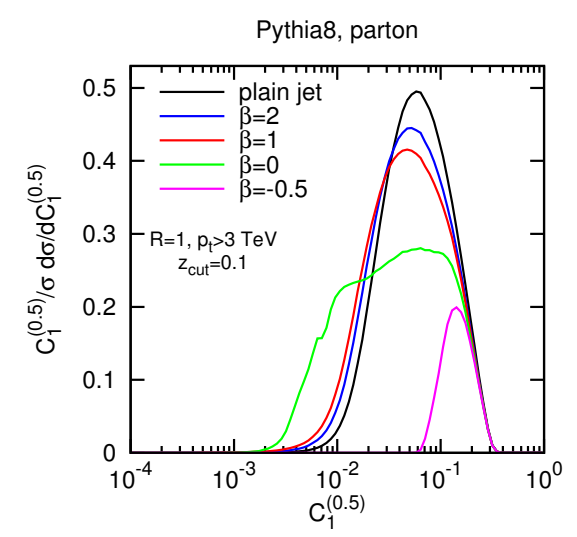

(e)

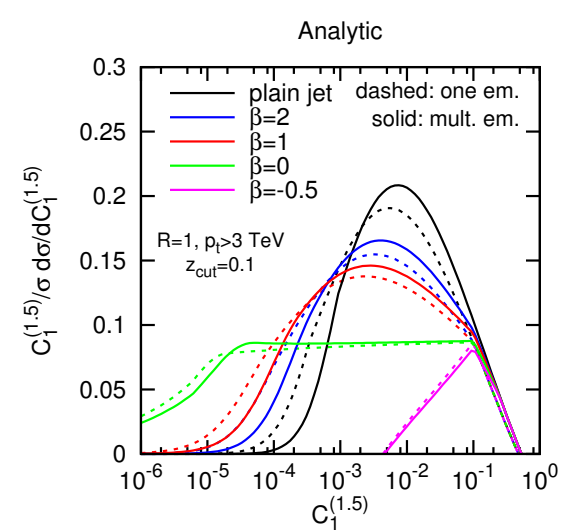

(b)

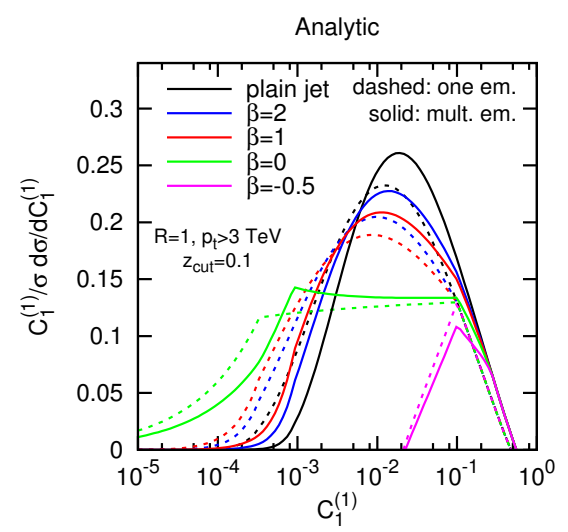

(d)

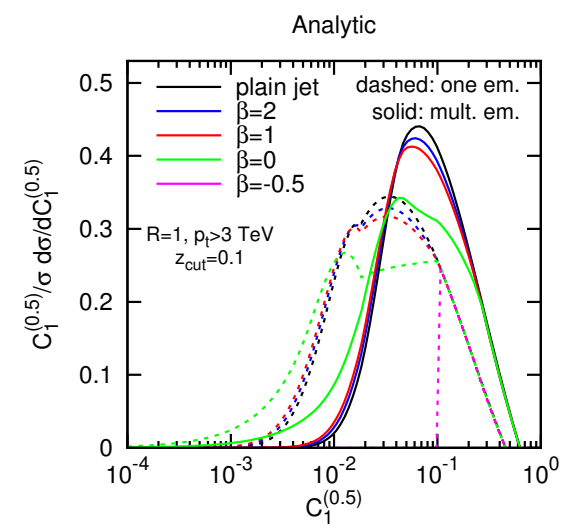

(f)

Figure 4: The energy correlation functions $C_{1}^{(\alpha)}$ with $\alpha=1.5,1,0.5$ (top to bottom) for quark-initiated jets. The plots on the left are obtained with PyтнIA 8, while the ones of the right are our MLL predictions (dashed) with multiple emissions included (solid). 
and our analytics. Moreover, we also note that the impact of multiple emissions, i.e. the difference between solid and dashed curves in figure $3 \mathrm{~b}$, decreases with $\beta$. It is perhaps surprising that we find worst agreement between analytics and Monte Carlo in the ungroomed (plain jet) case. However, one should keep in mind that although the two approximations are roughly of the same accuracy (MLL), Monte Carlo parton showers also partially contain many subleading effects. Using the results of refs. [92, 93], we have checked that subleading effects (like initial-state radiation and non-global logarithms) play a non-negligible role. Indeed, Pythia 8 is closer to the full NLL result than to the (less accurate) MLL plus multiple emissions one presented here. Because the action of soft drop is to remove largeangle soft radiation (e.g. initial state radiation and non-global logarithms), it is reassuring that our calculations for the finite $\beta$ soft-drop curves are indeed in better agreement with the parton shower.

In figure 4 , we compare our analytic resummation to the parton shower for $C_{1}^{(\alpha)}$ with $\alpha=1.5,1,0.5$. Again, the plots on the left are obtained with PyтніA 8 while the ones on the right are the MLL plus multiple emissions results. The same gross features seen with $\alpha=2$ are also present here, including the fact that the agreement between Monte Carlo and analytics is better with grooming than without. Overall, however, the agreement gets worse as $\alpha$ decreases. This is likely because, as seen in eq. (3.6), the expansion parameter is really $\alpha_{s} / \alpha$, so both logarithmically-enhanced and non-singular fixed-order corrections are more important at small $\alpha$. It is encouraging that the peak locations are still roughly the same in the analytic calculations and PYTHIA 8 results, even if the overall peak normalizations slightly differ. We note that the dashed curves in figure $4 \mathrm{f}$ have kinks; indeed all the curves in this section obtained from analytic calculations suffer from the same behavior, although this feature is not visible on the other plots. The position of the kink is $C_{1}^{(\alpha)}=\left(\frac{\mu_{\mathrm{NP}}}{p_{T} R_{0}}\right)^{\alpha}$ and it is a consequence of the way we freeze the running coupling at $\kappa=\mu_{\mathrm{NP}}$. As detailed in appendix A, this effect is beyond the accuracy of our calculation. Finally, in figures 4e and $4 \mathrm{f}$, note the sharp cutoff of the plots when $\alpha+\beta=0$, which can be understood from eq. (3.7). In figure $4 \mathrm{f}$, we only show the MLL result since fixed-order corrections are expected to be important and our treatment of multiple emissions effects in eq. (3.16) becomes singular when $\alpha+\beta=0$.

\section{Groomed jet radius}

Because the soft drop procedure is defined through declustering a $\mathrm{C} / \mathrm{A}$ branching tree, there is a well-defined and IRC-safe meaning to the groomed jet radius. Concretely, the groomed jet radius $R_{g}$ is the angle between the branches that first satisfy eq. (1.1), which is sensible because for a $\mathrm{C} / \mathrm{A}$ tree, all subsequent branches are separated by an angle less than $R_{g}$. From a practical perspective, $R_{g}$ is particularly interesting, since the groomed jet area is approximately $\pi R_{g}^{2}$. Thus, $R_{g}$ serves as a proxy for the sensitivity of the groomed jet to possible contamination from pileup $[124,125]$. 


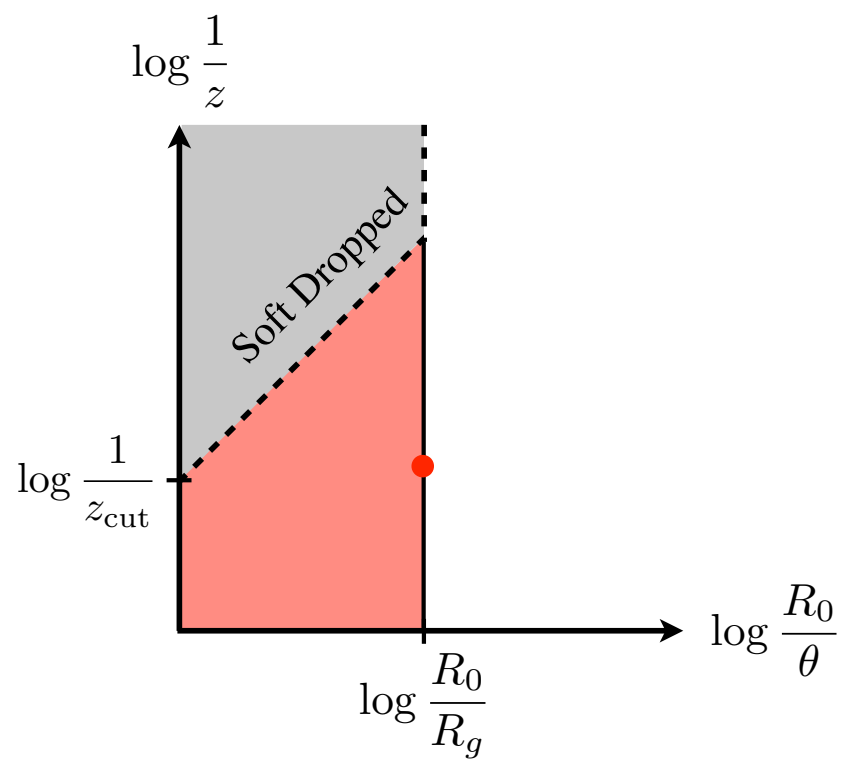

Figure 5: Phase space for emissions relevant for groomed jet radius $R_{g}$ in the $\left(\log \frac{1}{z}, \log \frac{R_{0}}{\theta}\right)$ plane. The soft dropped region is gray and the first emission satisfying the soft drop criteria is illustrated by the red dot. The forbidden emission region (the Sudakov exponent) is shaded in pink.

\subsection{Modified leading logarithmic approximation}

The calculation of the groomed jet radius to MLL accuracy follows much of the same logic as the $C_{1}^{(\alpha)}$ calculation in section 3.2. As illustrated in figure $5, R_{g}$ actually corresponds to a simpler phase space than $C_{1}^{(\alpha)}$. A given value of $R_{g}$ simply means that all emissions at angles larger than $R_{g}$ failed the soft drop criteria. Therefore, the $R_{g}$ distribution can be calculated by demanding that all emissions at angles larger than $R_{g}$ were groomed away. As explained in more detail in appendix B, this understanding translates into the following cumulative distribution for the groomed jet radius:

$$
\Sigma^{\text {radius }}\left(R_{g}\right)=\exp \left[-\int_{R_{g}}^{R_{0}} \frac{d \theta}{\theta} \int_{0}^{1} d z p_{i}(z) \frac{\alpha_{s}(\kappa)}{\pi} \Theta\left(z-z_{\text {cut }} \frac{\theta^{\beta}}{R_{0}^{\beta}}\right)\right],
$$

where the integral in the exponent again corresponds to vetoed emissions (i.e. the pink region in figure 5).

Besides the simpler phase space for one emission, $R_{g}$ is also simpler than $C_{1}^{(\alpha)}$ with respect to multiple emissions. In the case of $C_{1}^{(\alpha)}$, multiple emissions could contribute to the value of $C_{1}^{(\alpha)}$, but the MLL approximation effectively only considers the contribution from a single dominant emission. For $R_{g}$, though, once one emission satisfies the soft drop criteria, the jet radius is set, so multiple emissions do not contribute to this observable. We have also verified that non-global contributions are suppressed by $R_{g}$ for $\beta<\infty$, analogously to the energy correlation case. For these reasons, we believe that the expression in eq. (4.1) 
Pythia8, parton

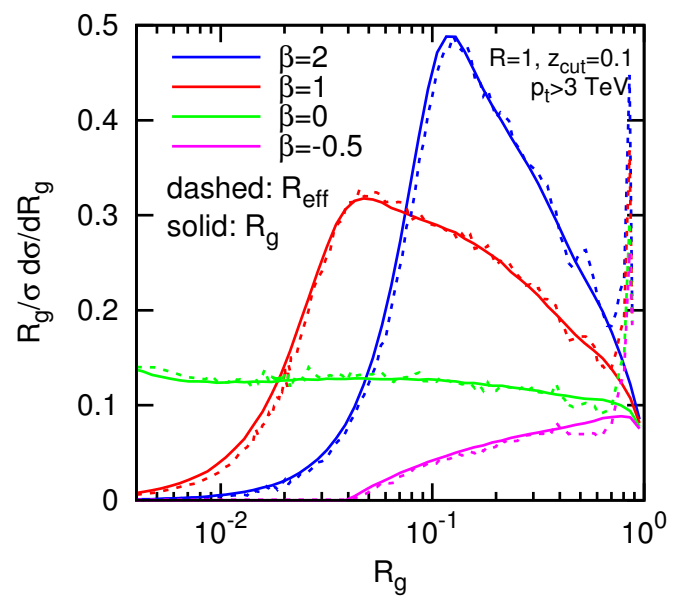

(a)

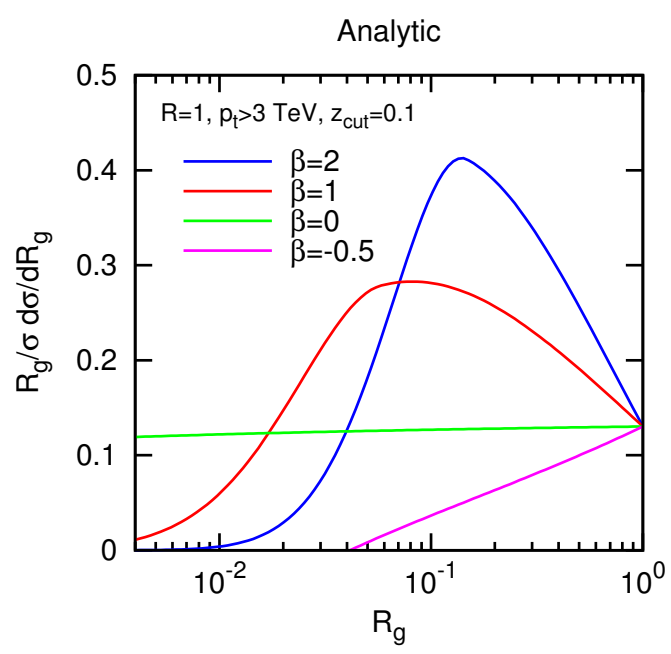

(b)

Figure 6: Comparison of the jet radius $R_{g}$ distribution extracted from PyтнIA 8 (left, solid) and inferred from the active area in PүтніA 8 using $R_{\text {eff }}=\sqrt{A_{\text {active }} / \pi \xi}$ (left, dashed), and computed to MLL accuracy (right). The original jet radius is set to be $R_{0}=1$ and the jets have an ungroomed energy of $3 \mathrm{TeV}$. The soft drop parameter is $z_{\text {cut }}=0.1$, while $\beta$ is varied.

is fully accurate to single-logarithmic level, ${ }^{8}$ though for consistency with the rest of this paper, we will only evaluate eq. (4.1) to MLL accuracy.

\subsection{Comparison to Monte Carlo}

There are two different ways one can define the groomed jet radius in Monte Carlo. The first method is to simply measure the $R_{g}$ value of the C/A branching that satisfies the soft drop condition. A second approach, more directly relevant for pileup mitigation, is to determine the effective radius of the groomed jet from its active area [108]. The active area of a jet is defined as the area over which infinitesimally soft particles are clustered into the jet. An effective jet radius $R_{\text {eff }}$ can then be defined from the groomed jet active area using:

$$
R_{\mathrm{eff}} \equiv\left(\frac{A_{\text {active }}}{\pi \xi}\right)^{1 / 2}
$$

where $A_{\text {active }}$ is the active jet area, and $\xi \simeq(1.16)^{2}$ accounts for the fact that a typical $\mathrm{C} / \mathrm{A}$ jet of radius $R_{0}$ has an average active area $\xi \pi R_{0}^{2}{ }^{9}$

\footnotetext{
${ }^{8}$ Strictly speaking, NLL accuracy requires evaluating the strong coupling at two loops, i.e. with $\beta_{1}$, in the CMW scheme [126].

${ }^{9}$ The numerical value for $\xi$ can be read from figure 8 in ref. [108]. Strictly speaking, this result is only valid for a jet made of two particles separated by $R_{0}$, with one of them much softer than the other. However, for $\mathrm{C} / \mathrm{A}$ jets, one expects that this would not vary much for more symmetric two-particle configurations (see e.g. ref. [125]).
} 


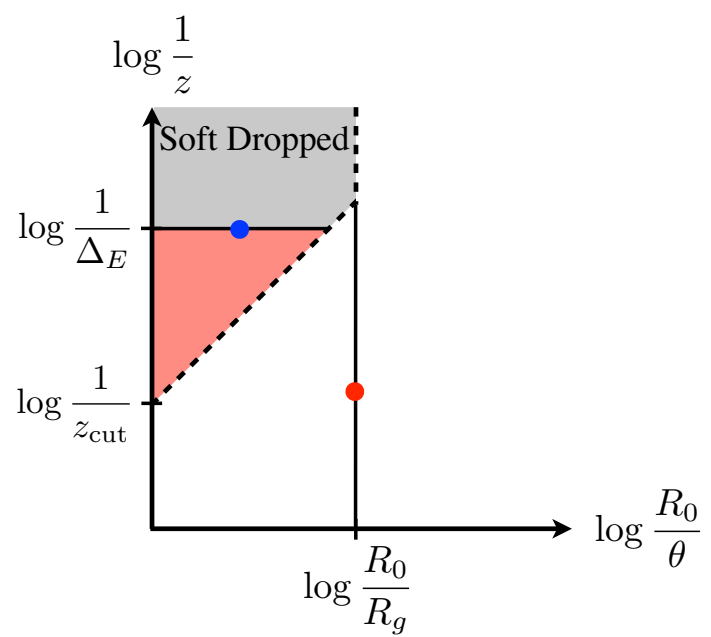

(a)

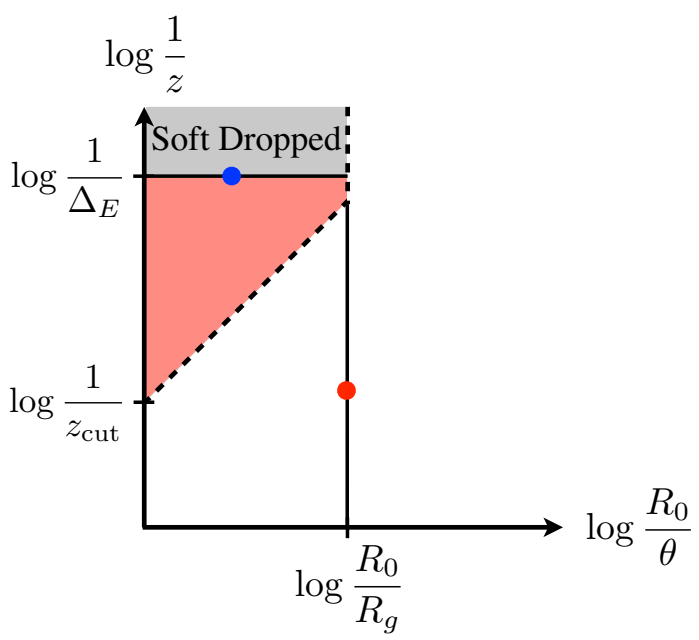

(b)

Figure 7: Phase space for emissions relevant for groomed jet energy loss $\Delta_{E}$ in the $\left(\log \frac{1}{z}, \log \frac{R_{0}}{\theta}\right)$ plane. The soft dropped region is gray/pink and the first emission satisfying the soft drop criteria is illustrated by the red dot, located at the groomed jet radius, $R_{g}$. The blue dot represents the leading contribution to $\Delta_{E}$, with subleading contributions above it. The location in angle of all soft dropped emissions is larger than $R_{g}$. The forbidden emission region for a given value of $R_{g}$ (the Sudakov exponent) is shaded in pink. The left (right) plot shows $\Delta_{E}$ larger (smaller) than $z_{\text {cut }}\left(R_{g} / R_{0}\right)^{\beta}$.

In figure 6a we show the $R_{g}$ and $R_{\text {eff }}$ distributions as measured on the same PYTHIA jet samples introduced in section 3.5. To obtain $R_{\text {eff }}$ in practice, we have computed the groomed jet area using active areas as implemented in FASTJET (v3), and we used a ghost area of 0.0005 and 10 repetitions in order to reach sufficiently small values of $R_{\text {eff }}$. With the $\xi$ offset factor, the two techniques give remarkably similar results, giving strong evidence that the groomed jet radius $R_{g}$ is an effective measure of pileup sensitivity. The main difference is the spike at $R_{\mathrm{eff}}=1 / \sqrt{\xi}$, corresponding to cases where the first C/A branching already satisfies the soft drop condition, yet typically with $R_{g}<1$. The nice reduction of the jet area even with mild grooming (e.g. $\beta=2$ ) suggests that soft drop should work well for pileup mitigation, but we leave a detailed study to future work.

In figure $6 \mathrm{~b}$, we show the MLL distribution from eq. (4.1). There is good qualitative agreement with PYтHIA for a range of angular exponents $\beta$, suggesting that our MLL calculation for $R_{g}$ captures the relevant physics effects present in the Monte Carlo simulation.

\section{Jet energy drop}

Our final analytic calculation is for the groomed jet energy. Unlike for many other grooming procedures, the energy of a soft-drop jet $(\beta>0)$ is an IRC safe observable and so can be computed in pQCD. In particular, we will study the fractional energy drop due to grooming 
$\Delta_{E}$ defined as

$$
\Delta_{E} \equiv \frac{E_{0}-E_{g}}{E_{0}},
$$

where $E_{0}$ is the energy of the jet before grooming and $E_{g}$ is the energy of the groomed jet. $\Delta_{E}$ can be interpreted as a measure of the fraction of the original jet's energy contained in soft wide-angle emissions. In the small $R_{0}$ limit, $\Delta_{E}$ is the same as the fractional $p_{T}$ loss, which is the more relevant quantity for non-central $(y \neq 0)$ jets in hadronic collisions.

\subsection{Modified leading logarithmic approximation}

At MLL order, the calculation of the $\Delta_{E}$ distribution is more subtle than for $C_{1}^{(\alpha)}$ or $R_{g}$. In the case of $C_{1}^{(\alpha)}$ and $R_{g}$, the Sudakov veto region was effectively determined by a single emission, and the multiple emissions effect for $C_{1}^{(\alpha)}$ could be included as a higher-order correction (see section 3.3).

For $\Delta_{E}$, the veto region depends crucially on two emissions, as illustrated in figure 7 . The energy drop due to grooming comes from large angle emissions that fail the soft drop condition. But to figure out which emissions are dropped, we first have to know which emission satisfied the soft drop condition, since that sets the groomed jet radius $R_{g}$. All emissions lying at angles greater than $R_{g}$ are removed by soft drop, but all emissions at angles less than $R_{g}$ are maintained. Thus, the energy drop depends both on the emission that sets $R_{g}$ and on the emissions that contributes to $\Delta_{E}$.

In practice, the easiest way to determine the $\Delta_{E}$ distribution is by computing the energy drop for a given value of $R_{g}$ and then integrating over the $R_{g}$ distribution. In equations, the cumulative distribution of $\Delta_{E}$ is given by

$$
\Sigma^{\text {energy-drop }}\left(\Delta_{E}\right)=\int_{0}^{R_{0}} d R_{g} \frac{d \Sigma^{\text {radius }}\left(R_{g}\right)}{d R_{g}} \widetilde{\Sigma}\left(R_{g}, \Delta_{E}\right)
$$

where $\widetilde{\Sigma}\left(R_{g}, \Delta_{E}\right)$ is the cumulative distribution of $\Delta_{E}$ for a given groomed jet radius $R_{g}$. The cumulative distribution $\Sigma^{\text {radius }}\left(R_{g}\right)$ was defined in eq. (4.1), and the derivative factor is needed to extract the differential cross section (i.e. the probability distribution) for $R_{g}$.

The details of the $\widetilde{\Sigma}\left(R_{g}, \Delta_{E}\right)$ calculation are presented in appendix C. The key is that this double cumulative distribution can be computed at logarithmic accuracy by summing over independent contributions at all orders:

$$
\begin{aligned}
\widetilde{\Sigma}\left(R_{g}, \Delta_{E}\right)= & \sum_{n=1}^{\infty}\left[\prod_{m=1}^{n} \int_{R_{g}}^{R_{0}} \frac{d \theta_{m}}{\theta_{m}} \int_{0}^{1} d z_{m} p_{i}\left(z_{m}\right) \frac{\alpha_{s}\left(\kappa_{m}\right)}{\pi} \Theta\left(\theta_{m}-\theta_{m+1}\right) \Theta\left(z_{\text {cut }} \frac{\theta_{m}^{\beta}}{R_{0}^{\beta}}-z_{m}\right)\right] \\
& \times \Theta\left(\Delta_{E}-\sum_{m=1}^{n} z_{m}\right) \exp \left[-\int_{R_{g}}^{R_{0}} \frac{d \theta}{\theta} \int_{0}^{1} d z p_{i}(z) \frac{\alpha_{s}(\kappa)}{\pi} \Theta\left(z_{\text {cut }} \frac{\theta^{\beta}}{R_{0}^{\beta}}-z\right)\right] \\
= & \int \frac{d \nu}{2 \pi i \nu} e^{\nu \Delta_{E}} e^{-R_{2}\left(R_{g}, \nu^{-1}\right)} .
\end{aligned}
$$

Here, we are accounting for the effect of multiple emissions (i.e. the sum over $m$ in the observable) by performing a Laplace transform, and the explicit integral over $\nu$ represents 
the inverse Laplace transform. The radiator function appearing in the exponent is

$$
R_{2}\left(R_{g}, \nu^{-1}\right)=\int_{R_{g}}^{R_{0}} \frac{d \theta}{\theta} \int_{0}^{1} d z p_{i}(z) \frac{\alpha_{s}(\kappa)}{\pi} \Theta\left(z_{\text {cut }} \frac{\theta^{\beta}}{R_{0}^{\beta}}-z\right)\left(1-e^{-\nu z}\right)
$$

which is a function of both the Laplace transform parameter $\nu$ and the groomed jet radius $R_{g}$.

\subsection{Sudakov safety for $\beta=0$}

As mentioned in section 2.2 , the groomed jet energy drop $\Delta_{E}$ is IRC safe only if $\beta>0$. In particular, the energy of a $\beta=0$ (mMDT) groomed jet is not an IRC safe quantity, since a measured value of energy does not require two well-separated hard prongs in the jet.

On the other hand, eq. (5.2) has a smooth $\beta \rightarrow 0$ limit, and therefore is still calculable (despite being IRC unsafe). Specifically, we are calculating the $\Delta_{E}$ distribution at a fixed groomed jet radius $R_{g}$, which forces a two-prong configuration. There is still an (IRC unsafe) singularity at $R_{g} \rightarrow 0$, but this is regulated by the Sudakov factor in the $R_{g}$ distribution. This property was referred to as "Sudakov safety" in ref. [105]. As we will now show, the way in which IRC unsafety but Sudakov safety manifests itself for $\Delta_{E}$ is rather peculiar.

The behavior of $\Delta_{E}$ for $\beta=0$ is easiest to study by computing the cumulative distribution of the energy drop at fixed coupling. We will also take the Laplace conjugate parameter $\nu \rightarrow \infty$ to suppress multiple emissions effects. This limit removes the inverse Laplace transform and turns the exponential factor in eq. (5.4) into the constraint that $z>\Delta_{E}$. We emphasize that the $\nu \rightarrow \infty$ limit is only taken to simplify the following discussion; the fixed-coupling energy loss distribution with the full multiple emissions effect exhibits the same properties.

At fixed-coupling, the cumulative distribution of the groomed jet radius is

$$
\begin{aligned}
\Sigma^{\text {radius }}\left(R_{g}\right) & \stackrel{\text { f.c. }}{=} \exp \left[-\frac{\alpha_{s}}{\pi} \int_{R_{g}}^{R_{0}} \frac{d \theta}{\theta} \int_{z_{\text {cut }}}^{1} d z p_{i}(z) \Theta\left(z-z_{\text {cut }} \frac{\theta^{\beta}}{R_{0}^{\beta}}\right)\right] \\
& \simeq \exp \left[-\frac{\alpha_{s}}{\pi} C_{i}\left(\beta \log ^{2} \frac{R_{0}}{R_{g}}-2 \log z_{\text {cut }} \log \frac{R_{0}}{R_{g}}+2 B_{i} \log \frac{R_{0}}{R_{g}}\right)\right]
\end{aligned}
$$

where we have ignored terms suppressed by positive powers of $z_{\text {cut }}$ and $\Delta_{E}$. The cumulative distribution of the energy drop at fixed groomed jet radius is

$$
\begin{aligned}
\widetilde{\Sigma}\left(R_{g}, \Delta_{E}\right) \stackrel{\text { f.c. }}{=} \exp \left[-\frac{\alpha_{s}}{\pi} \int_{R_{g}}^{R_{0}} \frac{d \theta}{\theta} \int_{\Delta_{E}}^{z_{\text {cut }}} d z p_{i}(z) \Theta\left(z_{\text {cut }} \frac{\theta^{\beta}}{R_{0}^{\beta}}-z\right)\right] \\
\simeq \Theta\left(z_{\text {cut }} \frac{R_{g}^{\beta}}{R_{0}^{\beta}}-\Delta_{E}\right) \exp \left[-\frac{\alpha_{s}}{\pi} C_{i}\left(2 \log \frac{z_{\text {cut }}}{\Delta_{E}} \log \frac{R_{0}}{R_{g}}-\beta \log ^{2} \frac{R_{0}}{R_{g}}\right)\right] \\
+\Theta\left(\Delta_{E}-z_{\text {cut }} \frac{R_{g}^{\beta}}{R_{0}^{\beta}}\right) \Theta\left(z_{\text {cut }}-\Delta_{E}\right) \exp \left[-\frac{\alpha_{s}}{\pi} \frac{C_{i}}{\beta} \log ^{2} \frac{z_{\text {cut }}}{\Delta_{E}}\right] .
\end{aligned}
$$


Plugging these expressions into eq. (5.2) in the $\nu \rightarrow \infty$ limit, we find the cumulative distribution of the groomed energy drop to be

$$
\Sigma^{\text {energy-drop }}\left(\Delta_{E}\right)=\frac{\log z_{\text {cut }}-B_{i}}{\log \Delta_{E}-B_{i}}+\frac{\pi \beta}{2 C_{i} \alpha_{s}\left(\log \Delta_{E}-B_{i}\right)^{2}}\left(1-e^{-2 \frac{\alpha_{S}}{\pi} \frac{C_{i}}{\beta} \log \frac{z_{\text {cut }}}{\Delta_{E}}\left(\log \frac{1}{\Delta_{E}}+B_{i}\right)}\right),
$$

for $\Delta_{E}<z_{\text {cut }}$. At this order, the cumulative distribution is constant for $\Delta_{E}>z_{\text {cut }}$.

The expression in eq. (5.7) has some fascinating properties. First, by expanding orderby-order in $\alpha_{s}$, we find

$$
\Sigma^{\text {energy-drop }}\left(\Delta_{E}\right)=1-\frac{\alpha_{s}}{\pi} \frac{C_{i}}{\beta} \log ^{2} \frac{z_{\mathrm{cut}}}{\Delta_{E}}+\mathcal{O}\left(\left(\frac{\alpha_{s}}{\beta}\right)^{2}\right) .
$$

Thus, the expansion in powers of the strong coupling is actually an expansion in $\alpha_{s} / \beta$, which diverges order-by-order in perturbation theory for $\beta \rightarrow 0$. Thus, as advertised, the energy drop distribution is not IRC safe for $\beta=0$. However, the $\beta \rightarrow 0$ limit of eq. (5.7) can be taken before expanding in $\alpha_{s}$. The $\beta \rightarrow 0$ limit yields the simple and surprising result

$$
\Sigma^{\text {energy-drop }}\left(\Delta_{E}\right)_{\beta=0}=\frac{\log z_{\text {cut }}-B_{i}}{\log \Delta_{E}-B_{i}}
$$

which is completely independent of $\alpha_{s}$ ! So while the strong coupling constant $\alpha_{s}$ was necessary to calculate $\Delta_{E}$, the leading behavior is independent of the value of $\alpha_{s}$.

We can attribute this behavior to the fact that $\Delta_{E}$ is a Sudakov safe observable for $\beta=0$. The singular region of phase space at $R_{g} \rightarrow 0$ is exponentially suppressed by the Sudakov factor in $\Sigma^{\text {radius }}\left(R_{g}\right)$. This exponential suppression balances the exponential increase in the number of groomed emissions in such a way that $\Delta_{E}$ is independent of $\alpha_{s}$. In fact, $\Delta_{E}$ is independent of the total color of the jet at fixed coupling, and only depends on the flavor of the jet through the subleading terms in the splitting functions $B_{i}$. When the running coupling is included, we will see that the dominant contribution to the $\Delta_{E}$ distribution is still independent of $\alpha_{s}$, with only weak dependence controlled by the QCD $\beta$-function.

\subsection{Non-global logarithms}

The ungroomed jet energy $E_{0}$ is clearly affected by non-global contributions, since emissions outside of the jet can radiate energy into the jet. Because the soft drop procedure removes soft wide-angle radiation, we expect that the groomed jet energy $E_{g}$ should have no nonglobal contributions. In principle, we could calculate the $E_{g}$ distribution directly to show the absence of non-global logarithms. In practice, though, it is hard to interpret the meaning of $E_{g}$ without invoking some reference energy scale. Here, we are using $E_{0}$ as a reference, which is not ideal since $E_{0}$ has non-global contributions. That said, we will find that the $E_{0}$ and $\Delta_{E}$ distributions have exactly the same non-global logarithms, implying that the $E_{g}$ distribution is wholly absent of them. 
Analogous to section 3.4, we can do a simple calculation of the non-global contribution to $\Delta_{E}$. At lowest order for a narrow jet of radius $R_{0}$, the non-global logarithms can be computed from

$$
\begin{aligned}
\frac{1}{\sigma_{0}} \frac{d \sigma^{\mathrm{NG}}}{d \Delta_{E}} & =4 C_{F} C_{A}\left(\frac{\alpha_{s}}{2 \pi}\right)^{2} \int \frac{d z_{1}}{z_{1}} \frac{d z_{2}}{z_{2}} \int d \theta_{1} d \theta_{2} \frac{4 \theta_{1} \theta_{2}}{\theta_{1}^{2}\left(\theta_{1}^{2}-\theta_{2}^{2}\right)} \Theta_{\mathrm{NG}} \Theta\left(z_{\text {cut }} \frac{\theta_{2}^{\beta}}{R_{0}^{\beta}}-z_{2}\right) \delta\left(\Delta_{E}-z_{2}\right) \\
& =\frac{2}{3} \pi^{2} C_{F} C_{A}\left(\frac{\alpha_{s}}{2 \pi}\right)^{2} \frac{\log \frac{1}{\Delta_{E}}}{\Delta_{E}}+\mathcal{O}\left(R_{0}^{2}, \frac{\Delta_{E}^{2 / \beta}}{z_{\text {cut }}^{2 / \beta}}\right) .
\end{aligned}
$$

This shows that non-global logarithms are not power-suppressed for the energy loss distribution regardless of $\beta$. Moreover, the coefficient of the non-global logarithms are the same for the ungroomed distribution $(\beta \rightarrow \infty)$ as for the groomed distribution (finite $\beta$ ). This implies that the groomed jet energy $E_{g}$ cannot contain any non-global logarithms.

Of course, to really verify this behavior, one would want to calculate the groomed jet energy distribution in a process with an additional scale. For example, one could study the associated production of a photon and a jet (i.e. $p p \rightarrow \gamma+j$ ) and use the photon momentum as a reference scale. In this example, we would expect $\left(p_{T g}-p_{T \gamma}\right) / p_{T \gamma}$ should be free of non-global logarithms.

\subsection{Comparison to Monte Carlo}

We conclude this section by comparing the fractional energy loss distribution between Pythia 8 to our MLL calculation, using the same jet samples as section 3.5. The comparison is shown in figure 8, with the Monte Carlo simulation on the left plot and our analytic calculation on the right. On the analytic plots, the solid (dashed) curves represent the result with (without) the inclusion of the multiple emission contributions to $\Delta_{E}$.

For $\beta>0$, there is good agreement between PyтнIA and our MLL analytics. For the IRC unsafe (but Sudakov safe) limit $\beta=0$, the agreement is fair in the region $\Delta_{E}<z_{\text {cut }}$. Note that $\beta=0$ has a large contribution from multiple emissions, but the structure of the inverse Laplace transform enforces that the MLL result cannot extend beyond $\Delta_{E}=z_{\text {cut }}$. In contrast, the PyтнiA distribution extends well beyond $z_{\text {cut }}$. This effect from multiple hard emissions contributing to $\Delta_{E}>z_{\text {cut }}$ is not captured by our resummation.

We can study the $\beta=0$ limit in PyтHIA to see whether the analytic predictions of section 5.2 are born out in Monte Carlo. In figure 9a, we show the $\Delta_{E}$ distribution for $\beta=0$ by artificially turning off the running coupling and setting the $\alpha_{s}$ value by hand. As discussed in eq. (5.9), the fixed-coupling analytic resummation does not depend on $\alpha_{s}$. Indeed, we see that the Monte Carlo results are fairly independent of the $\alpha_{s}$ value, and the behavior is well described by the analytic calculation. The same physical effect is seen in figure $9 \mathrm{~b}$, where the running coupling is restored but the distribution is shown for different choices of the minimum transverse momentum of the jet, which in turn probes different values of $\alpha_{s}$. We note that the curves differ very little from each other, suggesting that leading $\alpha_{s}$-independence of the $\beta=0$ result is robust. 


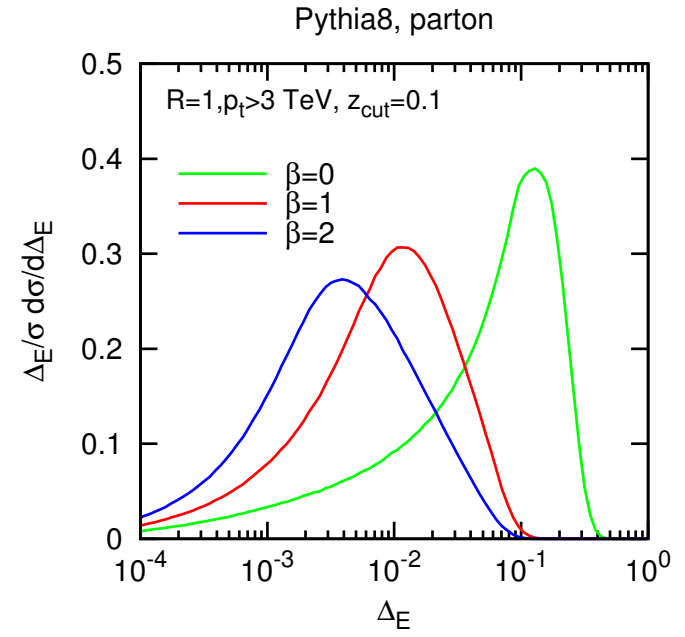

(a)

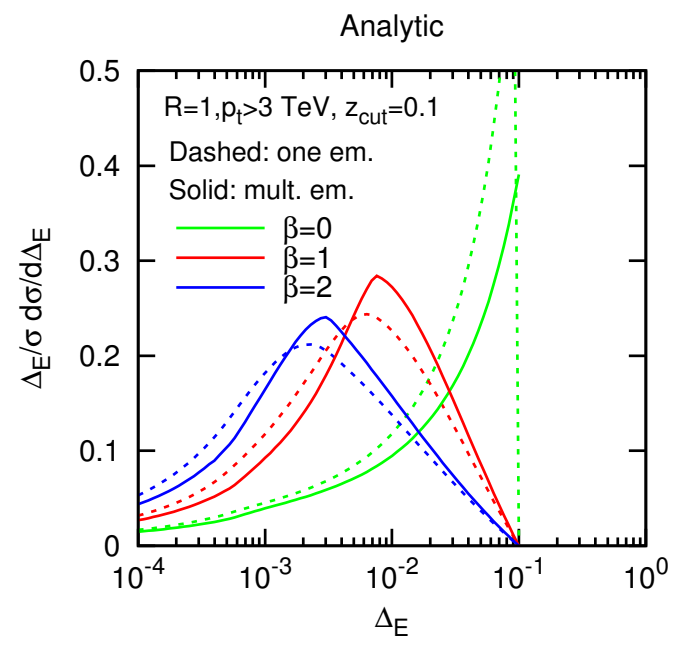

(b)

Figure 8: The distribution of groomed energy loss $\Delta_{E}$ in Pythia 8 (left) compared to our MLL calculation (right). In the MLL result, solid (dashed) corresponds to the distribution with (without) multiple emissions. The original jet radius is set to $R_{0}=1.0$ and the jets have an ungroomed energy of $3 \mathrm{TeV}$. The soft drop parameter $z_{\text {cut }}=0.1$ is fixed while $\beta$ is varied.

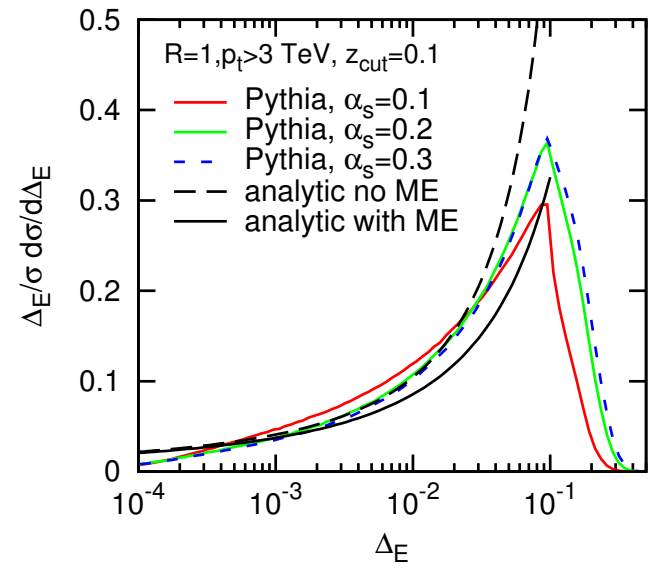

(a)

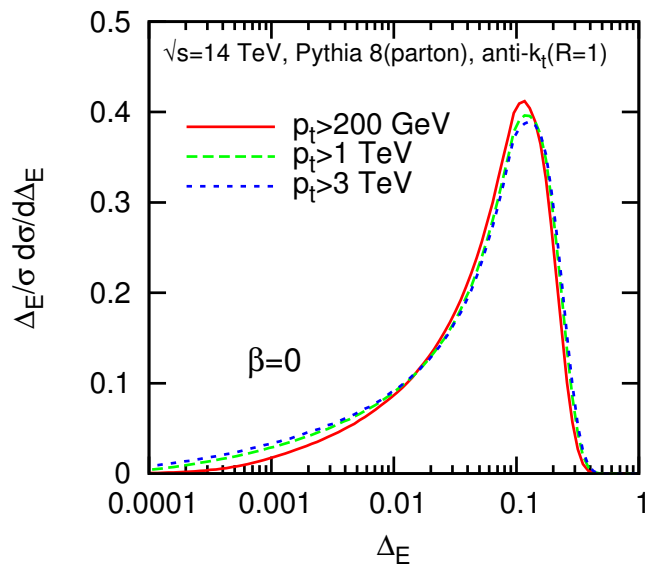

(b)

Figure 9: The dependence of the $\beta=0$ energy drop distribution on $\alpha_{s}$. On the left, we show Pyтhia results with fixed coupling compared to the fixed-coupling analytical prediction of eq. (5.9). On the right, we show the $\Delta_{E}$ distribution with running coupling at different values of the jet's transverse momentum. Both plots support the interpretation that the $\Delta_{E}$ distribution at $\beta=0$ is largely independent of $\alpha_{s}$. 


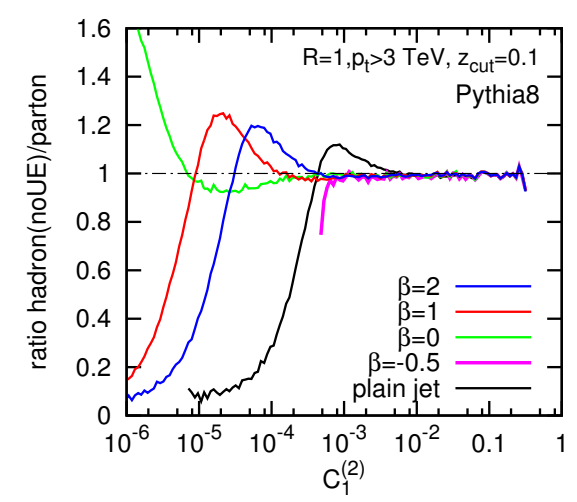

(a)

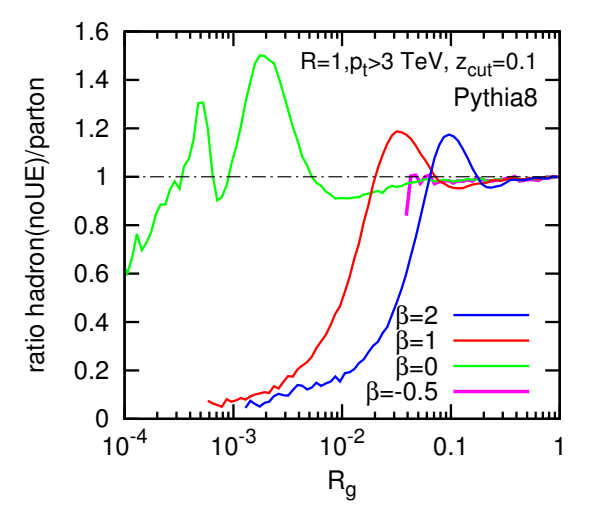

(c)

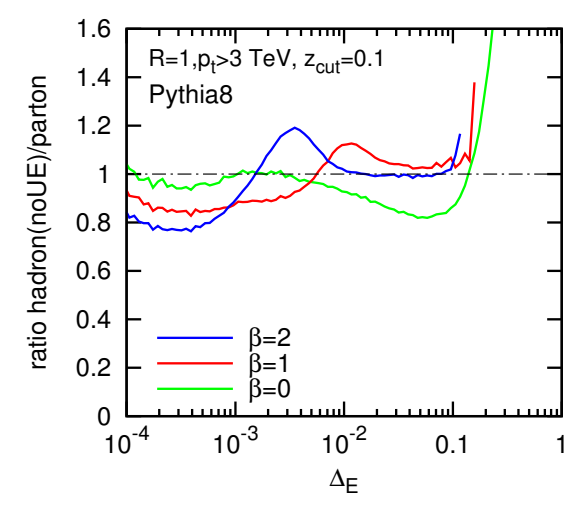

(e)

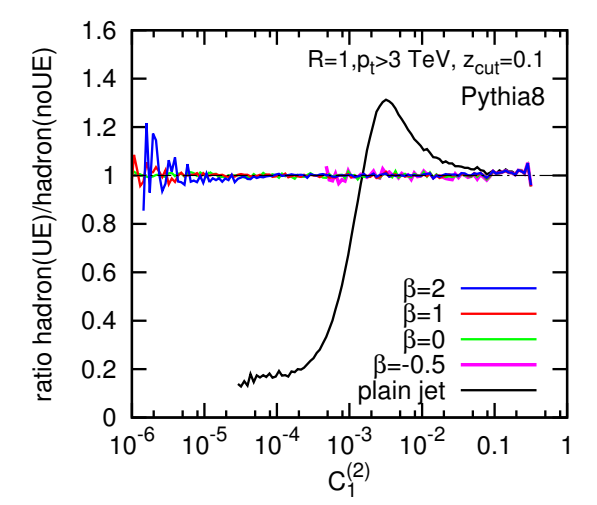

(b)

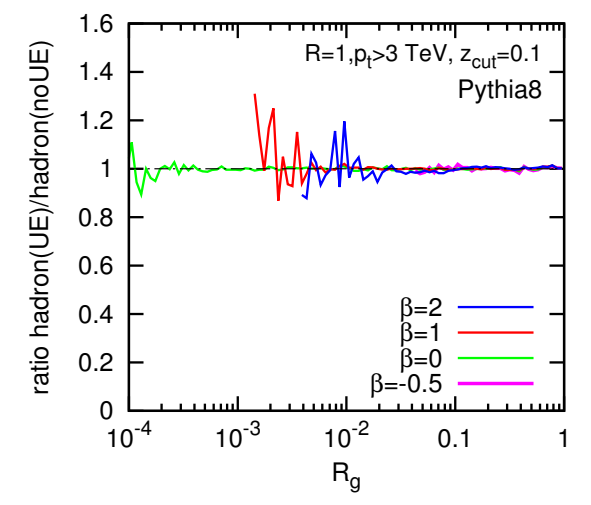

(d)

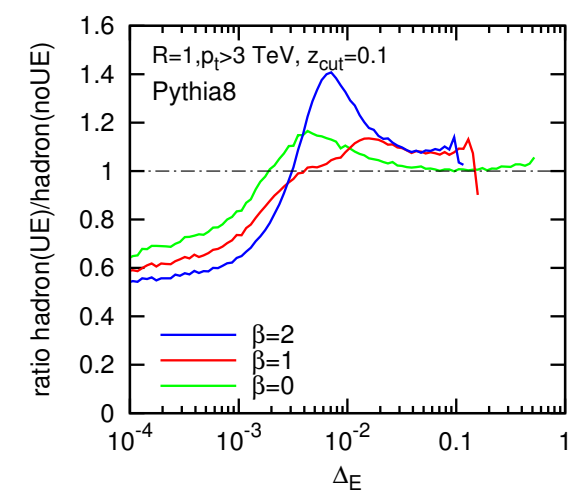

(f)

Figure 10: Effect of non-perturbative corrections on $C_{1}^{(\alpha=2)}$ (top), $R_{g}$ (middle), and $\Delta_{E}$ (bottom). The plots on the left show the ratio between hadron level and parton level predictions obtained with PYTHIA 8 (without UE). The plots on the right instead show the ratio of hadron-level results with and without UE. 


\section{Non-perturbative contributions}

In all of the above analytic calculations, we only considered the distributions generated by perturbative partons. In this section, we will do a brief Monte Carlo study to try to estimate the impact that non-perturbative effects from hadronization and UE can have on these distributions.

In figure 10, we show the effect of hadronization (left) and UE (right) for various observables considered in this paper. In the case of hadronization, we plot the ratio between the hadronic and partonic distributions obtained from Pythia 8. In the case of UE, we plot the ratio between the distributions with and without UE. Apart from including nonperturbative effects, the details of the analysis are the same as for the previous Monte Carlo studies.

We start by considering $C_{1}^{(\alpha)}$ for $\alpha=2$, i.e. similar to jet mass. The plot in figure 10a shows that soft drop declustering pushes the onset of hadronization corrections to smaller values of the observable compared to the ungroomed case (shown in black). As shown in figure 10b, soft drop has the remarkable ability to reduce the UE contribution to almost zero.

For the groomed jet radius distribution, the behavior of hadronization corrections in figure 10c is qualitatively similar to those seen for $C_{1}^{(\alpha)}$, with hadronization having a smaller effect for smaller values (and negative values) of $\beta$. The UE event contribution to $R_{g}$ in figure $10 \mathrm{~d}$ is also fairly small.

Finally, we show the effect of hadronization and UE corrections on the jet energy drop in figures 10e and 10f, respectively. Unlike for the previous distributions, the hadronization corrections are largest for $\beta=0$, which is likely related to the issue of IRC unsafety. For all values of $\beta$, the UE corrections are fairly large for $\Delta_{E}$. That said, because $\Delta_{E}$ is defined in terms of both the groomed energy $E_{g}$ and the ungroomed energy $E_{0}$, it is hard to know whether these effects are caused mainly by $E_{g}$ or $E_{0}$. We suspect that $E_{g}$ is rather robust to UE effects, and the dominant change is really from distortions of the reference $E_{0}$ value.

\section{Boosted $W$ tagging with soft drop}

Thus far, we have studied the analytic properties of soft drop declustering and argued that it can be a successful grooming technique for $\beta>0$. For $\beta<0$, soft drop acts like a tagger which identifies jets with hard two-prong structures. Here, we investigate the performance of soft drop in tagging mode by doing a brief study of boosted $W$ tagging.

To have a source of fat $W$ and QCD jets, we generated $W W$ and dijet samples with Pythia 8 for $14 \mathrm{TeV}$ proton-proton collisions, including all non-perturbative effects from tune $4 \mathrm{C}$. As in the previous Monte Carlo studies in this paper, we start from anti- $k_{t}$ jets with $R_{0}=1$, this time keeping only jets with $p_{T} \geq p_{T \text { min }}$ and rapidity $|y|<4$. These samples of $W$ (signal) and QCD (background) jets are then groomed/tagged using soft drop with various values of $\beta$ and $z_{\text {cut }}$, and we define the efficiency/mistag rates from the fraction of selected jets after soft drop with groomed masses in the $W$ window $[70 \mathrm{GeV}, 90 \mathrm{GeV}]$. 


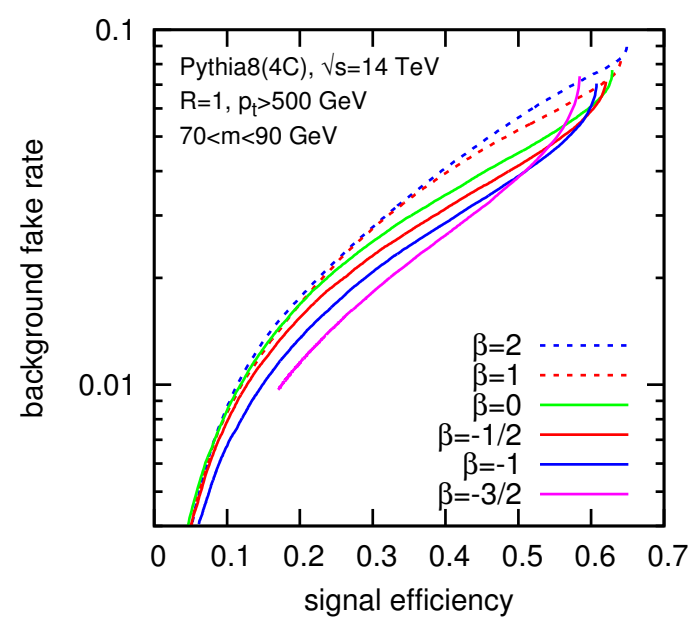

(a)

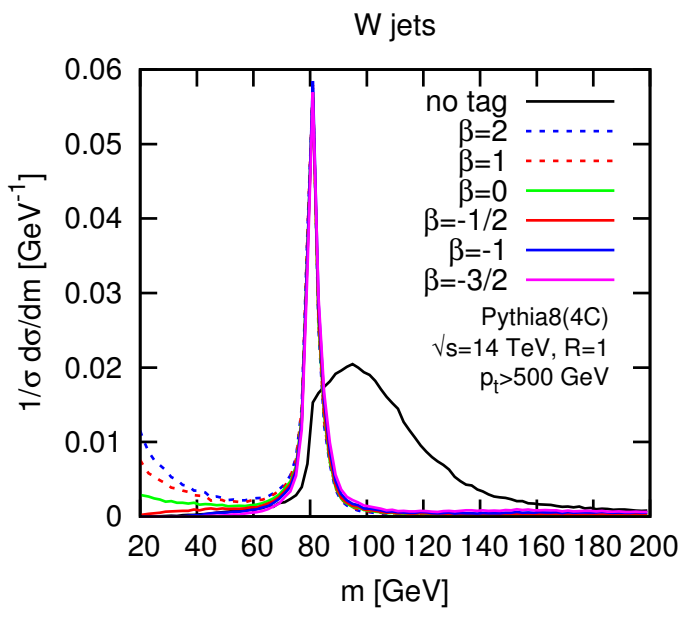

(c)

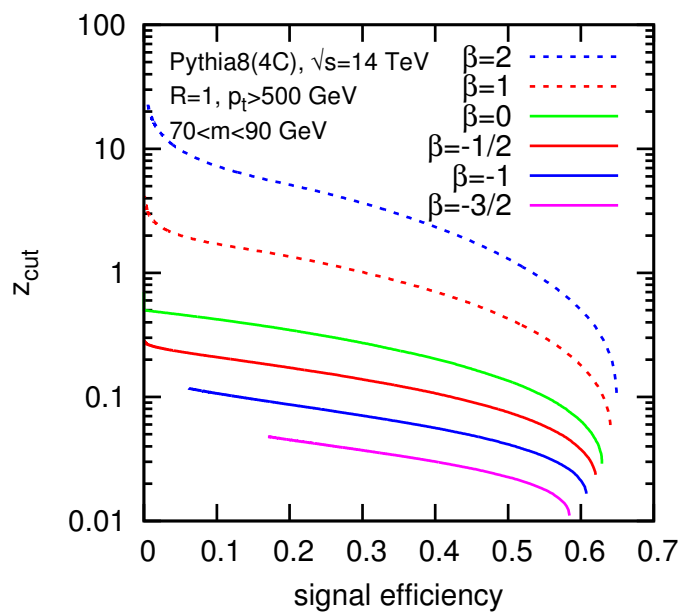

(b)

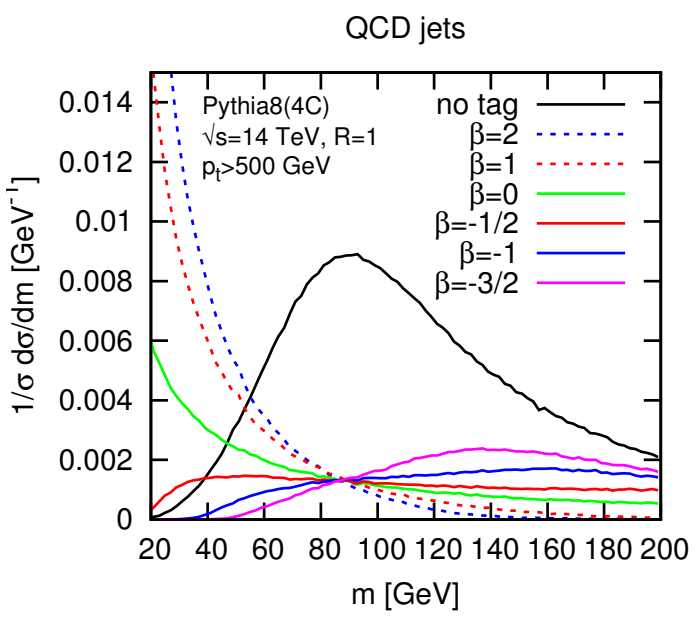

(d)

Figure 11: Performance of soft drop as a boosted $W$ tagger. Top left: signal efficiency versus background mistag for jets with $p_{T}>500 \mathrm{GeV}$. Each curve is obtained by fixing the value of $\beta$, sweeping the value of $z_{\text {cut }}$, and counting jets with groomed mass in the range [70 GeV, $90 \mathrm{GeV}]$. Top right: Values of $z_{\text {cut }}$ for as a function of the efficiency, for given $\beta$. Bottom: mass distribution of signal (left) and background (right) jets before and after soft drop. For each curve, the value of $\beta$ is shown in the legend, while the value of $z_{\text {cut }}$ is the one that gives a $35 \%$ signal efficiency. 


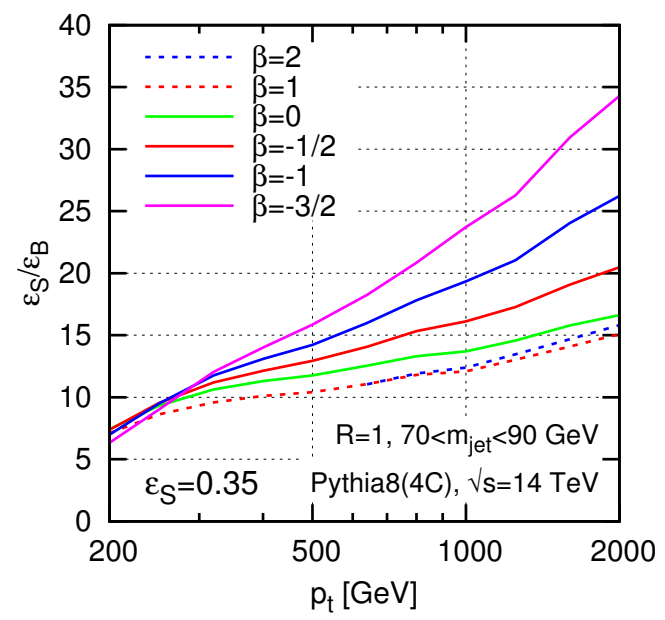

Figure 12: Ratio of signal-to-background efficiency as a function of the minimum jet $p_{T}$ for fixed signal efficiency of $35 \%$.

The results of this study are presented in figures 11 and 12 . In figure 11a, we fix $p_{T \text { min }}=500 \mathrm{GeV}$ and study the efficiency/mistag rates for fixed $\beta$, sweeping $z_{\text {cut }}$. The values of $z_{\text {cut }}$ found as a function of the efficiency are shown in figure 11b. As initially expected, negative values of $\beta$ (i.e. tagging mode) tend to have a higher performance than positive values (i.e. grooming mode). Note also that the $z_{\text {cut }}$ values for $\beta<0$ fall in the more reasonable range of $z_{\text {cut }} \lesssim 1$, whereas $z_{\text {cut }} \gtrsim 1$ is needed to obtain comparable performance for $\beta>0$.

In figures $11 \mathrm{c}$ and $11 \mathrm{~d}$, we show the mass distributions of signal and background jets with $p_{T}>500 \mathrm{GeV}$ after soft-drop, for different values of $\beta$ and choosing the value of $z_{\text {cut }}$ that correspond to $35 \%$ signal efficiency. Regarding the signal, all values of $\beta$ yield a nice narrow mass distributions around $m_{W}$. Without soft drop, the background in this $p_{T}$ window happens to (accidentally) have a mass peak around $m_{W}$, but as desired, the soft-dropped background mass distributions are pushed away from the signal region.

Finally, in figure 12, we study the ratio of signal-to-background efficiency as a function of $p_{T \text { min }}$ (at fixed $35 \%$ signal efficiency). Negative values of $\beta$ continue to have a higher performance, especially at large $p_{T}$, essentially because of a stronger Sudakov suppression of the background at fixed signal efficiency. The overall performance is comparable to other $W$ tagging methods, with percent-level mistag rates at $35 \%$ efficiency.

The original mass-drop prescription from ref. [6] also involves a filtering step. There, the filtering radius was taken as $\min \left(R_{g} / 2,0.3\right)$ with $R_{g}$ defined as in section 4 , and the three hardest subjets were kept. However, applying filtering on soft-dropped jets is not necessarily beneficial. For example, at large $p_{T}$ and for $\beta<0$, the action of the soft drop is such that the background peaks at a value of the mass larger than the $W$ mass. In this case, filtering would slightly shift this peak to smaller masses, increasing the background rates. On the other hand, we should add that in a situation with pileup, filtering or some similar form of grooming might also be needed in order to improve the resolution on the 
signal peak. We leave a detailed study of the interplay between pileup mitigation and boosted object tagging to future work.

\section{Conclusions}

In this paper, we introduced the soft drop declustering procedure. Soft drop generalizes the mMDT procedure by incorporating an angular exponent $\beta$, and simplifies mMDT by removing the mass drop condition. True to its name, the soft drop procedure drops wideangle soft radiation from a jet, though for $\beta \leq 0$ it can also drop collinear radiation. To demonstrate the analytic behavior of soft drop declustering, we calculated three distributions to MLL accuracy (while also including multiple emissions): the energy correlation functions $C_{1}^{(\alpha)}$, the groomed jet radius $R_{g}$, and the jet energy drop $\Delta_{E}$. Two particularly interesting analytic features are the smooth turn off of non-global logarithms for $C_{1}^{(\alpha)}$ in the $\beta \rightarrow 0$ limit and the approximate $\alpha_{s}$-independence of the jet energy drop distribution for $\beta=0$.

Beyond our analytic calculations, we studied the performance of soft drop in two other contexts. We used a Monte Carlo study to estimate the impact of non-perturbative effects, and found that soft drop reduces the impact of hadronization and UE corrections on $C_{1}^{(\alpha)}$ compared to ungroomed case. We also used a Monte Carlo study to demonstrate that soft drop with $\beta<0$ can act as an effective tagger for boosted $W$ bosons.

One area for future study is the behavior of soft drop as a pileup mitigation tool. We have seen that soft drop yields small values of $R_{g}$ and hence small jet areas, so one might expect that soft drop would have similar pileup performance to trimming [53, 69, 76, 89]. Like trimming, soft drop declustering with $\beta>0$ is an all-purpose grooming procedure, in the sense that the grooming procedure does not veto jets (unlike a tagger), and the groomed version of an (otherwise) IRC safe observable is still IRC safe. ${ }^{10}$ Both trimming and soft drop have two parameters. In the case of trimming, they are the energy fraction threshold $f_{\text {cut }}$ and the subjet radius $R_{\text {sub }}$. In the case of soft drop, they are the soft drop threshold $z_{\text {cut }}$ and the angular exponent $\beta$. The $f_{\text {cut }}$ and $z_{\text {cut }}$ parameters play a similar role, since they control how aggressive the grooming procedure is and also define the transition points in, e.g., the $C_{1}^{(\alpha)}$ distribution.

However, there is a qualitative difference between $R_{\mathrm{sub}}$ and $\beta$ which is likely to be phenomenologically relevant. At fixed values of the jet mass, harder jets become narrower jets. The radius parameter $R_{\text {sub }}$ sets a fixed angular scale, such that narrower jets are effectively groomed less (see the discussion in ref. [59]). In contrast, $\beta$ sets a scaling relation between energies and angles, such that the amount of grooming decreases only gradually as the jets become more narrow. The extreme limit of $\beta=0$ is where approximately the same fraction of energy is groomed away regardless of the initial jet energy (see eq. (5.9)). Thus, we expect that soft drop could potentially have better performance than trimming at

\footnotetext{
${ }^{10}$ This is in contrast to the $\beta=0$ (mMDT) limit, which has to be run in tagging mode to obtain an IRC safe groomed jet energy distribution. Of course, we have argued that $\beta=0$ soft-dropped distributions are still Sudakov safe.
} 
higher energies and luminosities. Of course, this assumes that detectors are able to resolve angular scales smaller than the typical $R_{\text {sub }} \simeq 0.2$.

Finally, in a more speculative vein, one might wonder whether the soft drop procedure could be applied on an event-wide basis instead of jet-by-jet as considered here. In the case of trimming, there is a suitable generalization [127] such that the trimming criteria can be imposed without needing to first cluster an event into jets. In the case of mass drop, there are ways to sew together different jet multiplicities to impose a kind of event-wide mass drop condition [43]. If the soft drop condition in eq. (1.1) could be applied on an eventwide basis, this could help address many of the numerous complications associated with soft radiation and allow analyses to focus on the more tractable collinear physics of jets.

\section{Acknowledgments}

We thank the organizers of the ESI Program on Jets and QFT and of Boost 2013 for stimulating workshops during which this project was started. We acknowledge useful discussions with Mrinal Dasgupta, Gavin Salam and Jeff Tseng. S.M. would like to thank IPhT Saclay for hospitality during the course of this project. A.L. and J.T. are supported by the U.S. Department of Energy (DOE) under cooperative research agreement DE-FG02-05ER-41360. J.T. is also supported by the DOE Early Career research program DE-FG02-11ER-41741 and by a Sloan Research Fellowship from the Alfred P. Sloan Foundation. S.M. is supported by the UK Science \& Technology Facilities Council (STFC). G.S. is supported by the French Agence Nationale de la Recherche, under grant ANR-10-CEXC-009-01 and by the EU ITN grant LHCPhenoNet, PITN-GA-2010-264564.

\section{A Details of energy correlation calculation}

We present the details of the calculation of the soft-drop energy correlation function $\left(C_{1}^{(\alpha)}\right.$ with $\alpha>0$ ) to MLL accuracy. Thus, we consider the independent emission of $n$ collinear gluons within a jet. For each splitting $m$, the scale of the (one-loop) coupling is chosen at the relative transverse momentum scale $\kappa_{m}=z_{m} \theta_{m} p_{T \text { jet }}$. This is sufficient to capture logarithmic accuracy we seek in this study (for a more detailed discussion, see the extensive literature on event-shape and jet-mass resummation, e.g. [114-116]).

The above undestanding translates into

$$
\begin{aligned}
\Sigma\left(C_{1}^{(\alpha)}\right)= & \sum_{n=0}^{\infty} \frac{1}{n !} \prod_{m=1}^{n} \int \frac{d \theta_{m}}{\theta_{m}} \int d z_{m} p_{i}\left(z_{m}\right) \frac{\alpha_{s}\left(\kappa_{m}\right)}{\pi}\left[\Theta\left(z_{\text {cut }}\left(\frac{\theta_{m}}{R_{0}}\right)^{\beta}-z_{m}\right)\right. \\
& \left.+\Theta\left(z_{m}-z_{\text {cut }}\left(\frac{\theta_{m}}{R_{0}}\right)^{\beta}\right) \Theta\left(C_{1}^{(\alpha)}-z_{m}\left(\frac{\theta_{m}}{R_{0}}\right)^{\alpha}\right)-1\right] \Theta\left(R_{0}-\theta_{m}\right) \\
= & \sum_{n=0}^{\infty} \frac{(-1)^{n}}{n !} \prod_{m=1}^{n} \int \frac{d \theta_{m}}{\theta_{m}} \int d z_{m} p_{i}\left(z_{m}\right) \frac{\alpha_{s}\left(\kappa_{m}\right)}{\pi}\left[\Theta\left(z_{m}\left(\frac{\theta_{m}}{R_{0}}\right)^{\alpha}-C_{1}^{(\alpha)}\right)\right. \\
& \left.\times \Theta\left(z_{m}-z_{\text {cut }}\left(\frac{\theta_{m}}{R_{0}}\right)^{\beta}\right)\right] \Theta\left(R_{0}-\theta_{m}\right) .
\end{aligned}
$$


To MLL accuracy, the resummed result is then

$$
\Sigma\left(C_{1}^{(\alpha)}\right)=e^{-R\left(C_{1}^{(\alpha)}\right)}
$$

where the radiator is given by the integral of the one-loop contribution over the allowed phase-space:

$$
R\left(C_{1}^{(\alpha)}\right)=\frac{1}{\alpha} \int_{C_{1}^{(\alpha)}}^{1} \frac{d c}{c} \int_{\max \left(c, z_{\mathrm{cut}} \frac{\alpha}{\alpha+\beta} \frac{\beta}{c^{\alpha+\beta}}\right)}^{1} d z p_{i}(z) \frac{\alpha_{s}(\kappa)}{\pi}
$$

The reduced splitting functions $p_{i}$, with $i=q, g$ are given by

$$
\begin{aligned}
& p_{q}(z)=C_{F} \frac{1+(1-z)^{2}}{z} \\
& p_{g}(z)=C_{A}\left[2 \frac{1-z}{z}+z(1-z)+\frac{T_{R} n_{f}}{C_{A}}\left(z^{2}+(1-z)^{2}\right)\right] .
\end{aligned}
$$

Note that for small enough values of energy fractions $z$ and angular distances $\theta$, the argument of the coupling in eq. (A.3) can approach the non-perturbative region. Thus, we introduce a prescription in order to evaluate the integrals down to these low scales. We decide to freeze the coupling below a non-perturbative scale $\mu_{\mathrm{NP}}$ :

$$
\alpha_{s}(\kappa)=\alpha_{s}^{1-\text { loop }}(\kappa) \Theta\left(\kappa-\mu_{\mathrm{NP}}\right)+\alpha_{s}^{1-\mathrm{loop}}\left(\mu_{\mathrm{NP}}\right) \Theta\left(\mu_{\mathrm{NP}}-\kappa\right)
$$

where $\alpha_{s}^{1-\text { loop }}(\kappa)$ is the usual one-loop expression for the strong coupling, i.e. its running is evaluated with $\beta_{0}$ only:

$$
\alpha_{s}^{1-\operatorname{loop}}(\kappa)=\frac{\alpha_{s}(Q)}{1+2 \alpha_{s}(Q) \beta_{0} \log \frac{\kappa}{Q}}
$$

Our results are expressed in terms of $\alpha_{s}=\alpha_{s}\left(R_{0} p_{T}\right)$ and we use $\alpha_{s}\left(m_{Z}\right)=0.12, n_{f}=5$, and $\mu_{\mathrm{NP}}=1 \mathrm{GeV}$ throughout this paper.

In the $\left(\log \frac{1}{z}, \log \frac{R_{0}}{\theta}\right)$ plane of figure 2 , the boundary between perturbative and nonperturbative regions is given by

$$
\kappa=\mu_{\mathrm{NP}} \quad \Rightarrow \quad \log \frac{1}{z}=\log \frac{1}{\tilde{\mu}}-\log \frac{R_{0}}{\theta}
$$

where we have introduced $\tilde{\mu}=\frac{\mu_{\mathrm{NP}}}{p_{T} R_{0}}$. Thus, according to eq. (A.5), below this straight line, the one-loop running coupling is evaluated at the relative transverse momentum $\kappa$, while above this line it is frozen at $\mu_{\mathrm{NP}}$.

The explicit form for the non-perturbative region of result depends on the relation between the slope of lines of constant $C_{1}^{(\alpha)}$, which is controlled by $\alpha$, and the slope of the boundary between perturbative and non-perturbative regime, given by eq. (A.7). We also assume $\beta \geq 0$ for simplicity and discuss the case $\beta<0$ in the end. 
For $\alpha>1$ we find

$$
\begin{aligned}
R\left(C_{1}^{(\alpha)}\right) \stackrel{C_{1}^{(\alpha)} \geq z_{\mathrm{cut}}}{=} & \frac{C_{i}}{2 \pi \alpha_{s} \beta_{0}^{2}}\left[\frac{\mathrm{W}(1-\lambda)}{\alpha-1}-\frac{\alpha \mathrm{W}\left(1-\frac{1}{\alpha} \lambda\right)}{\alpha-1}-2 \alpha_{s} \beta_{0} B_{i} \log \left(1-\frac{1}{\alpha} \lambda\right)\right] \\
z_{\mathrm{cut}}^{\frac{1-\alpha}{1+\beta} \tilde{\mu}^{\frac{\alpha+\beta}{1+\beta}}=C_{1}^{(\alpha)}<z_{\mathrm{cut}}} \frac{C_{i}}{2 \pi \alpha_{s} \beta_{0}^{2}}\left[-\frac{\mathrm{W}\left(1-\lambda_{c}\right)}{1+\beta}-\frac{\alpha \mathrm{W}\left(1-\frac{1}{\alpha} \lambda\right)}{\alpha-1}-2 \alpha_{s} \beta_{0} B_{i} \log \left(1-\frac{1}{\alpha} \lambda\right)\right. & \\
& \left.+\frac{\alpha+\beta}{(\alpha-1)(1+\beta)} \mathrm{W}\left(1-\frac{1+\beta}{\alpha+\beta} \lambda-\frac{\alpha-1}{\alpha+\beta} \lambda_{c}\right)\right] \\
\tilde{\mu}^{\alpha}<C_{1}^{(\alpha)}<z_{\mathrm{cut}}^{\frac{1-\alpha}{1+\beta}} \tilde{\mu}^{\frac{\alpha+\beta}{1+\beta}} & \frac{C_{i}}{2 \pi \alpha_{s} \beta_{0}^{2}}\left[-\frac{\mathrm{W}\left(1-\lambda_{c}\right)}{1+\beta}-\frac{\alpha \mathrm{W}\left(1-\frac{1}{\alpha} \lambda\right)}{\alpha-1}-2 \alpha_{s} \beta_{0} B_{i} \log \left(1-\frac{1}{\alpha} \lambda\right)\right. \\
& \left.-\frac{1+\log \left(1-\lambda_{\mu}\right)}{(\alpha-1)(1+\beta)}\left((\alpha-1) \lambda_{c}+(1+\beta) \lambda-(\alpha+\beta) \lambda_{\mu}\right)\right) \\
& \left.+\frac{\alpha+\beta}{(\alpha-1)(1+\beta)} \mathrm{W}\left(1-\lambda_{\mu}\right)\right]+\frac{C_{i} \alpha_{s}\left(\mu_{\mathrm{NP}}\right)}{\pi} \mathcal{F}_{1}(L) \\
& \frac{C_{i}}{2 \pi \alpha_{s} \beta_{0}^{2}}\left[-\frac{\mathrm{W}\left(1-\lambda_{c}\right)}{1+\beta}-\frac{\beta \mathrm{W}\left(1-\lambda_{\mu}\right)}{1+\beta}-2 \alpha_{s} \beta_{0} B_{i} \log \left(1-\lambda_{\mu}\right)\right. \\
& \left.-\frac{1+\log \left(1-\lambda_{\mu}\right)}{(1+\beta)}\left(\lambda_{c}+\beta \lambda_{\mu}\right)\right]+\frac{C_{i} \alpha_{s}\left(\mu_{\mathrm{NP}}\right)}{\pi}\left[\mathcal{F}_{1}\left(\alpha L_{\mu}\right)\right. \\
& \left.+\left(\frac{L}{\alpha}-L_{\mu}\right)\left(\frac{2 \alpha}{\alpha+\beta} L_{c}+2 B_{i}+\frac{\beta}{\alpha+\beta}\left(L+\alpha L_{\mu}\right)\right)\right]
\end{aligned}
$$

while for $\alpha<1$ we have ${ }^{11}$

$$
\begin{aligned}
& R\left(C_{1}^{(\alpha)}\right) \stackrel{C_{1}^{(\alpha)} \geq z_{\text {cut }}}{=} \frac{C_{i}}{2 \pi \alpha_{s} \beta_{0}^{2}}\left[\frac{\mathrm{W}(1-\lambda)}{\alpha-1}-\frac{\alpha \mathrm{W}\left(1-\frac{1}{\alpha} \lambda\right)}{\alpha-1}-2 \alpha_{s} \beta_{0} B_{i} \log \left(1-\frac{1}{\alpha} \lambda\right)\right] \\
& \tilde{\mu}^{\alpha}<C_{1}^{(\alpha)}<z_{\text {cut }} \frac{C_{i}}{2 \pi \alpha_{s} \beta_{0}^{2}}\left[-\frac{\mathrm{W}\left(1-\lambda_{c}\right)}{1+\beta}-\frac{\alpha \mathrm{W}\left(1-\frac{1}{\alpha} \lambda\right)}{\alpha-1}-2 \alpha_{s} \beta_{0} B_{i} \log \left(1-\frac{1}{\alpha} \lambda\right)\right. \\
& \left.+\frac{\alpha+\beta}{(\alpha-1)(1+\beta)} \mathrm{W}\left(1-\frac{1+\beta}{\alpha+\beta} \lambda-\frac{\alpha-1}{\alpha+\beta} \lambda_{c}\right)\right] \\
& z_{\mathrm{cut}}^{\frac{1-\alpha}{1+\beta}} \stackrel{\frac{\alpha+\beta}{1+\beta}}{=}<C_{1}^{(\alpha)}<\tilde{\mu}^{\alpha} \frac{C_{i}}{2 \pi \alpha_{s} \beta_{0}^{2}}\left[-\frac{\mathrm{W}\left(1-\lambda_{c}\right)}{1+\beta}-\frac{\alpha \mathrm{W}\left(1-\lambda_{\mu}\right)}{\alpha-1}+\frac{\lambda-\alpha \lambda_{\mu}}{\alpha-1}\left(1+\log \left(1-\lambda_{\mu}\right)\right)\right. \\
& \left.-2 \alpha_{s} \beta_{0} B_{i} \log \left(1-\lambda_{\mu}\right)+\frac{\alpha+\beta}{(\alpha-1)(1+\beta)} \mathrm{W}\left(1-\frac{1+\beta}{\alpha+\beta} \lambda-\frac{\alpha-1}{\alpha+\beta} \lambda_{c}\right)\right] \\
& +\frac{C_{i} \alpha_{s}\left(\mu_{\mathrm{NP}}\right)}{\pi}\left(\frac{L}{\alpha}-L_{\mu}\right)\left(\frac{L-\alpha L_{\mu}}{1-\alpha}+2 B_{i}\right) \\
& C_{1}^{(\alpha)}<z_{\text {cut }}^{\frac{1-\alpha}{1+\beta}} \tilde{\mu}^{\frac{\alpha+\beta}{1+\beta}} \frac{C_{i}}{2 \pi \alpha_{s} \beta_{0}^{2}}\left[-\frac{\mathrm{W}\left(1-\lambda_{c}\right)}{1+\beta}-\frac{\beta \mathrm{W}\left(1-\lambda_{\mu}\right)}{1+\beta}-\left(\frac{\lambda_{c}+\beta \lambda_{\mu}}{1+\beta}\right)\right. \\
& \left.\times\left(1+\log \left(1-\lambda_{\mu}\right)\right)-2 \alpha_{s} \beta_{0} B_{i} \log \left(1-\lambda_{\mu}\right)\right] \\
& +\frac{C_{i} \alpha_{s}\left(\mu_{\mathrm{NP}}\right)}{\pi}\left[\mathcal{F}_{2}(L)+\frac{(1-\alpha)\left(\beta L_{\mu}+L_{c}\right)\left(2(1+\beta) B_{i}+\beta L_{\mu}+L_{c}\right)}{\alpha(1+\beta)^{2}}\right] .
\end{aligned}
$$

\footnotetext{
${ }^{11}$ For definiteness we consider the case $z_{\text {cut }}>\tilde{\mu}^{\alpha}$.
} 
In the above expressions, we have introduced $\mathrm{W}(x)=x \log x$ and

$$
\begin{aligned}
\mathcal{F}_{1}(L)= & \frac{\left((1+\beta) L-(\alpha+\beta) L_{\mu}+(\alpha-1) L_{c}\right)^{2}}{(\alpha-1)(1+\beta)(\alpha+\beta)}, \\
\mathcal{F}_{2}(L)= & \frac{(1+\beta) L-(\alpha+\beta) L_{\mu}+(\alpha-1) L_{c}}{\alpha(1+\beta)^{2}(\alpha+\beta)} \\
& \times\left(\beta(\alpha+\beta) L_{\mu}+2 B_{i}(1+\beta)(\alpha+\beta)+L_{c}(2 \alpha+\alpha \beta+\beta)+\beta(1+\beta) L\right) .
\end{aligned}
$$

Here, $C_{i}$ is the color of the jet appropriate for quarks $\left(C_{q}=C_{F}\right)$ or gluons $\left(C_{g}=C_{A}\right)$. $B_{i}$ describes the contribution to the cross section from collinear logarithms: $B_{q}=-3 / 4$ for quark jets and $B_{g}=-\frac{11}{12}+\frac{n_{f}}{6 C_{A}}$ for gluon jets, where $n_{f}$ is the number of active quark flavors. We have also introduced

$$
\begin{array}{lll}
L=\log \left(1 / C_{1}^{(\alpha)}\right) & L_{c}=\log \left(1 / z_{\text {cut }}\right) & L_{\mu}=\log (1 / \tilde{\mu}), \\
\lambda=2 \alpha_{s} \beta_{0} L & \lambda_{c}=2 \alpha_{s} \beta_{0} L_{c} & \lambda_{\mu}=2 \alpha_{s} \beta_{0} L_{\mu} .
\end{array}
$$

Moreover, it can be easily checked that the limit $\alpha \rightarrow 1$ is perfectly safe because the two non-perturbative transition points coincide and therefore one non-perturbative region disappears.

We note that expression for the running coupling with non-perturbative freezing eq. (A.5) has a discontinuous first derivative at $\kappa=\mu_{\mathrm{NP}}$. To our logarithmic accuracy, this behavior is reflected into a discontinuity of the second derivative of the radiator at $C_{1}^{(\alpha)}=\tilde{\mu}^{\alpha}$, which in turns causes a kink in the spectrum. The difference between rightand left- second derivatives of the radiator at $C_{1}^{(\alpha)}=\tilde{\mu}^{\alpha}$ is

$$
c=\left(\frac{\alpha_{s}\left(\mu_{\mathrm{NP}}\right)}{\pi}\right)^{2} \frac{4 \pi \beta_{0} C_{F} B_{i}}{\alpha^{2}}
$$

which is a contribution beyond the accuracy of our calculation. This effect is bigger for smaller $\alpha$, and for the case $\alpha=0.5$ in figure $4 \mathrm{f}$, the non-perturbative transition point $C_{1}^{(\alpha)}=\tilde{\mu}^{\alpha}$ occurs in the vicinity of the Sudakov peak. Clearly, this is an artefact of our choice of an abrupt freezing of the coupling in the non-perturbative region. One could imagine to alter eq. (A.5) in such a way that it smoothly interpolates between running and fixed coupling (as done, for example, with scale profiling [128]). Alternatively, one could add an appropriate (subleading) term to the radiator in the region $C_{1}^{(\alpha)}<\tilde{\mu}^{\alpha}$. However, we have decided not to introduce an ad-hoc prescription and, in this paper, we present results obtained from our MLL calculations (plus eventually multiple-emission effects), with the freezing of coupling previously discussed.

As already mentioned, the results for the resummed exponent have been obtained assuming $\beta>0$. It is clear from the expressions above that the $\beta \rightarrow 0$ limit is perfectly safe. Indeed for $\beta=0$ the result considerably simplifies and one obtains the mMDT singlelogarithmic distribution. Moreover, the same results also hold for the $\beta<0$ case, provided that $C_{1}^{(\alpha)}>z_{\text {cut }}^{\alpha /|\beta|}$, which is the minimum allowed value for the energy correlation function. For $C_{1}^{(\alpha)}<z_{\text {cut }}^{\alpha \| \beta \mid}$, the radiator freezes at $R\left(z_{\text {cut }}^{\alpha /|\beta|}\right)$ and consequently the differential distribution vanishes. 
Finally, the above results are also sufficient to compute the multiple-emission contributions described in section 3.3, which simply involve the derivative, as defined in eq. (3.16), of the radiator functions derived in this appendix.

\section{B Details of jet radius calculation}

Here, we present the details of the calculation of the cumulative cross section of the jet radius after soft drop declustering. Because we are interested in the behavior of soft drop as a grooming procedure, we only consider $\beta>0$. As presented in section 4 , the cumulative resummed cross section can be computed from the sum over emissions as

$$
\begin{aligned}
\Sigma^{\text {radius }}\left(R_{g}\right)=\sum_{n=1}^{\infty} \frac{1}{n !} & \prod_{m=1}^{n}\left[\int_{R_{g}}^{R_{0}} \frac{d \theta_{m}}{\theta_{m}} \int_{0}^{1} d z_{m} p_{i}\left(z_{m}\right) \frac{\alpha_{s}\left(\kappa_{m}\right)}{\pi} \Theta\left(z_{\text {cut }} \frac{\theta_{m}^{\beta}}{R_{0}^{\beta}}-z_{m}\right)\right] \\
& \times e^{-\int_{R_{g}}^{R_{0}} \frac{d \theta}{\theta} \int_{0}^{1} d z p_{i}(z) \frac{\alpha_{s}(\kappa)}{\pi}} \\
= & e^{-R_{1}\left(R_{g}\right)}
\end{aligned}
$$

where the exponent $R_{1}\left(R_{g}\right)$ is given by

$$
R_{1}\left(R_{g}\right)=\int_{R_{g}}^{R_{0}} \frac{d \theta}{\theta} \int_{0}^{1} d z p_{i}(z) \frac{\alpha_{s}(\kappa)}{\pi} \Theta\left(z-z_{\text {cut }} \frac{\theta^{\beta}}{R_{0}^{\beta}}\right) .
$$

The evaluation of the integrals proceed analogously to the case of the energy correlation case described in detail in appendix A. In this case, the radiator is found to be

$$
\begin{aligned}
& R_{1}\left(R_{g}\right) \stackrel{R_{g}>R_{g}^{(0)}}{=} \frac{C_{i}}{2 \pi \alpha_{s} \beta_{0}^{2}}\left[-\mathrm{W}\left(1-\lambda_{g}\right)-\frac{\mathrm{W}\left(1-\lambda_{c}\right)}{1+\beta}+\frac{1}{1+\beta} \mathrm{W}\left(1-\lambda_{c}-(1+\beta) \lambda_{g}\right)\right. \\
& \left.-2 \alpha_{s} \beta_{0} B_{i} \log \left(1-\lambda_{g}\right)\right] \\
& \mu R_{0}<R_{g}<R_{g}^{(0)} \frac{C_{i}}{2 \pi \alpha_{s} \beta_{0}^{2}}\left[-\mathrm{W}\left(1-\lambda_{g}\right)-\frac{\mathrm{W}\left(1-\lambda_{c}\right)}{1+\beta}+\frac{1-\lambda_{c}-(1+\beta) \lambda_{g}}{1+\beta} \log \left(1-\lambda_{\mu}\right)\right. \\
& \left.+\frac{\lambda_{\mu}-\lambda_{c}-(1+\beta) \lambda_{g}}{1+\beta}-2 \alpha_{s} \beta_{0} B_{i} \log \left(1-\lambda_{g}\right)\right] \\
& +\frac{C_{i} \alpha_{s}\left(\mu_{\mathrm{NP}}\right)}{\pi} \frac{1}{1+\beta}\left[(1+\beta) L_{g}+L_{c}-L_{\mu}\right]^{2} \\
& \stackrel{R_{g}<\mu}{=} R_{0} \quad \frac{C_{i}}{2 \pi \alpha_{s} \beta_{0}^{2}}\left[-\frac{\mathrm{W}\left(1-\lambda_{c}\right)}{1+\beta}-\frac{\lambda_{c}+\beta}{1+\beta} \log \left(1-\lambda_{\mu}\right)-\frac{\lambda_{c}+\beta \lambda_{\mu}}{1+\beta}\right. \\
& \left.-2 \alpha_{s} \beta_{0} B_{i} \log \left(1-\lambda_{\mu}\right)\right] \\
& +\frac{C_{i} \alpha_{s}\left(\mu_{\mathrm{NP}}\right)}{\pi}\left[\frac{1}{1+\beta}\left(\beta L_{\mu}+L_{c}\right)^{2}+\left(L_{g}-L_{\mu}\right)\left(\beta L_{g}+\beta L_{\mu}+2 L_{c}+2 B_{i}\right)\right]
\end{aligned}
$$

with $R_{g}^{(0)}=R_{0}\left(\tilde{\mu} / z_{\text {cut }}\right)^{1 /(1+\beta)}$ and $L_{g}=\log \left(R_{0} / R_{g}\right), \lambda_{g}=2 \alpha_{s} \beta_{0} L_{g}$. 


\section{Details of energy drop calculation}

Here, we present the details of the calculation of the cumulative cross section for the fractional energy drop from soft drop declustering. Because we are interested in behavior of soft drop as a grooming procedure, we only consider $\beta>0$. As opposed to the calculations previously described, for the energy drop distribution we also consider the effect of multiple emissions. The resummed cumulative distribution is most easily written at fixed groomed jet radius $R_{g}$. The resulting expression is then integrated over all possible values of $R_{g}$ :

$$
\Sigma^{\text {energy-drop }}\left(\Delta_{E}\right)=\int_{0}^{R_{0}} d R_{g} \frac{d \Sigma^{\text {radius }}\left(R_{g}\right)}{d R_{g}} \int \frac{d \nu}{2 \pi i \nu} e^{\nu \Delta_{E}} e^{-R_{2}\left(R_{g}, \nu^{-1}\right)},
$$

and $R_{2}\left(R_{g}, \nu\right)$ is the radiator function:

$$
R_{2}\left(R_{g}, \nu^{-1}\right)=\int_{R_{g}}^{R_{0}} \frac{d \theta}{\theta} \int_{0}^{1} d z p_{i}(z) \frac{\alpha_{s}(\kappa)}{\pi} \Theta\left(z_{\text {cut }} \frac{\theta^{\beta}}{R_{0}^{\beta}}-z\right)\left(1-e^{-\nu z}\right),
$$

In order to capture the single-logarithmic terms in eq. (C.2) arising from multiple emissions we can make the following simplification [114-116]

$$
R_{2}\left(R_{g}, \nu^{-1}\right) \simeq \bar{R}_{2}\left(R_{g}, \nu^{-1}\right)+\gamma_{E} \bar{R}_{2}^{\prime}\left(R_{g}, \nu^{-1}\right)
$$

where

$$
\bar{R}_{2}\left(R_{g}, \nu^{-1}\right)=2 \int_{R_{g}}^{R_{0}} \frac{d \theta}{\theta} \int_{\nu^{-1}}^{1} \frac{d z}{z} \frac{\alpha_{s}(\kappa)}{\pi} \Theta\left(z_{\text {cut }} \frac{\theta^{\beta}}{R_{0}^{\beta}}-z\right),
$$

$\gamma_{E}$ is the Euler-Mascheroni constant and $\bar{R}_{2}^{\prime}\left(R_{g}, \nu^{-1}\right)$ is the logarithmic derivative of $\bar{R}_{2}$ with respect to $\nu$. Moreover, note that we were able to drop the finite contributions to the splitting function $p_{i}(z)$ because for small values of $z_{\text {cut }}$, there are no logarithms from hard collinear emission.

The inverse Laplace transform in eq. (C.1) can be done to single logarithmic accuracy in $\nu$, also, by expanding $\nu$ about a fixed value $\nu_{0}$. Doing this, the inverse Laplace transform becomes

$$
\int \frac{d \nu}{2 \pi i \nu} e^{\nu \Delta_{E}} e^{-R_{2}\left(R_{g}, \nu^{-1}\right)}=\frac{\left(\nu_{0} \Delta_{E}\right)^{-\bar{R}_{2}^{\prime}\left(R_{g}, \nu_{0}^{-1}\right)}}{\Gamma\left(1+\bar{R}_{2}^{\prime}\left(R_{g}, \nu_{0}^{-1}\right)\right)} e^{-\bar{R}_{2}\left(R_{g}, \nu_{0}^{-1}\right)-\gamma_{E} \bar{R}_{2}^{\prime}\left(R_{g}, \nu_{0}^{-1}\right)} .
$$

To minimize the logarithms, we choose $\nu_{0}=\Delta_{E}^{-1}$ and so the cumulative distribution of the groomed jet energy drop becomes

$$
\Sigma^{\text {energy-drop }}\left(\Delta_{E}\right)=\int_{0}^{R_{0}} d R_{g} \frac{d \Sigma^{\text {radius }}\left(R_{g}\right)}{d R_{g}} \frac{e^{-\gamma_{E} \bar{R}_{2}^{\prime}\left(R_{g}, \Delta_{E}\right)}}{\Gamma\left(1+\bar{R}_{2}^{\prime}\left(R_{g}, \Delta_{E}\right)\right)} e^{-\bar{R}_{2}\left(R_{g}, \Delta_{E}\right)},
$$

The evaluation of the integrals with running coupling proceeds in the same way as discussed for the energy correlation and groomed-jet radius distributions. We first obtain the energy drop cumulative distribution at fixed $R_{g}$ and then numerically integrate of $R_{g}$. 
The radiator $\bar{R}_{2}$ is better described in three regions of $R_{g}$. First, for $R_{g}>R_{0}\left(\tilde{\mu} / z_{\text {cut }}\right)^{1 /(1+\beta)}$, we find

$$
\begin{aligned}
& \bar{R}_{2}\left(\Delta_{E}\right) \stackrel{\Delta_{E}>z_{\mathrm{cut}}\left(R_{g} / R_{0}\right)^{\beta}}{=} \frac{C_{i}}{2 \pi \alpha_{s} \beta_{0}^{2}}\left[\frac{\mathrm{W}\left(1-\lambda_{c}\right)}{1+\beta}-\mathrm{W}\left(1-\lambda_{E}\right)+\frac{\beta}{1+\beta} \mathrm{W}\left(1+\frac{\lambda_{c}-(1+\beta) \lambda_{E}}{\beta}\right)\right] \\
& \tilde{\mu} R_{0} / R_{g}<\Delta_{E} \leq z_{\mathrm{cut}}\left(R_{g} / R_{0}\right)^{\beta} \frac{C_{i}}{2 \pi \alpha_{s} \beta_{0}^{2}}\left[\frac{\mathrm{W}\left(1-\lambda_{c}\right)}{1+\beta}-\mathrm{W}\left(1-\lambda_{E}\right)-\frac{\mathrm{W}\left(1-\lambda_{c}-(1+\beta) \lambda_{g}\right)}{1+\beta}\right. \\
& \left.+\mathrm{W}\left(1-\lambda_{g}-\lambda_{E}\right)\right] \\
& \tilde{\mu}<\Delta_{E}<\stackrel{\tilde{\mu}}{=} R_{0} / R_{g} \frac{C_{i}}{2 \pi \alpha_{s} \beta_{0}^{2}}\left[\frac{\mathrm{W}\left(1-\lambda_{c}\right)}{1+\beta}-\mathrm{W}\left(1-\lambda_{E}\right)-\frac{\mathrm{W}\left(1-\lambda_{c}-(1+\beta) \lambda_{g}\right)}{1+\beta}\right. \\
& \left.+\left(1-\lambda_{g}-\lambda_{E}\right) \log \left(1-\lambda_{\mu}\right)+\left(\lambda_{\mu}-\lambda_{g}-\lambda_{E}\right)\right] \\
& +\frac{C_{i} \alpha_{s}\left(\mu_{\mathrm{NP}}\right)}{\pi}\left(L_{g}+L_{E}-L_{\mu}\right)^{2} \\
& \stackrel{\Delta_{E}<\tilde{\mu}}{=} \quad \frac{C_{i}}{2 \pi \alpha_{s} \beta_{0}^{2}}\left[\frac{\mathrm{W}\left(1-\lambda_{c}\right)}{1+\beta}-\frac{\mathrm{W}\left(1-\lambda_{c}-(1+\beta) \lambda_{g}\right)}{1+\beta}-\lambda_{g} \log \left(1-\lambda_{\mu}\right)-\lambda_{g}\right] \\
& +\frac{C_{i} \alpha_{s}\left(\mu_{\mathrm{NP}}\right)}{\pi} L_{g}\left(L_{g}+2 L_{E}-2 L_{\mu}\right) \text {. }
\end{aligned}
$$

Then, for $\left(\tilde{\mu} / z_{\text {cut }}\right)^{1 / \beta}<R_{g} / R_{0}<\left(\tilde{\mu} / z_{\text {cut }}\right)^{1 /(1+\beta)}$, we find

$$
\begin{aligned}
& \bar{R}_{2}\left(\Delta_{E}\right) \stackrel{\Delta_{E}>\Delta_{E}^{(0)}}{=} \quad \frac{C_{i}}{2 \pi \alpha_{s} \beta_{0}^{2}}\left[\frac{\mathrm{W}\left(1-\lambda_{c}\right)}{1+\beta}-\mathrm{W}\left(1-\lambda_{E}\right)+\frac{\beta}{1+\beta} \mathrm{W}\left(1+\frac{\lambda_{c}-(1+\beta) \lambda_{E}}{\beta}\right)\right] \\
& z_{\mathrm{cut}}\left(R_{g} / R_{0}\right)^{\beta}<\Delta_{E}<\Delta_{E}^{(0)} \frac{C_{i}}{2 \pi \alpha_{s} \beta_{0}^{2}}\left[\frac{\mathrm{W}\left(1-\lambda_{c}\right)}{1+\beta}-\mathrm{W}\left(1-\lambda_{E}\right)+\frac{\beta+\lambda_{c}-(1+\beta) \lambda_{E}}{1+\beta} \log \left(1-\lambda_{\mu}\right)\right. \\
& \left.+\frac{\lambda_{c}+\beta \lambda_{\mu}-(1+\beta) \lambda_{E}}{1+\beta}\right]+\frac{C_{i} \alpha_{s}\left(\mu_{\mathrm{NP}}\right)}{\pi} \frac{1+\beta}{\beta}\left(L_{E}-\frac{L_{c}+\beta L_{\mu}}{1+\beta}\right)^{2} \\
& \tilde{\mu}<\Delta_{E}<z_{\text {cut }}\left(R_{g} / R_{0}\right)^{\beta} \frac{C_{i}}{2 \pi \alpha_{s} \beta_{0}^{2}}\left[\frac{\mathrm{W}\left(1-\lambda_{c}\right)}{1+\beta}-\mathrm{W}\left(1-\lambda_{E}\right)+\frac{\beta+\lambda_{c}-(1+\beta) \lambda_{E}}{1+\beta} \log \left(1-\lambda_{\mu}\right)\right. \\
& \left.+\frac{\lambda_{c}+\beta \lambda_{\mu}-(1+\beta) \lambda_{E}}{1+\beta}\right] \\
& +\frac{C_{i} \alpha_{s}\left(\mu_{\mathrm{NP}}\right)}{\pi}\left[\left(L_{E}+L_{g}-L_{\mu}\right)^{2}-\frac{\left(L_{c}+(1+\beta) L_{g}-L_{\mu}\right)^{2}}{1+\beta}\right] \\
& \stackrel{\Delta_{E}<\tilde{\mu}}{=} \quad \frac{C_{i}}{2 \pi \alpha_{s} \beta_{0}^{2}}\left[\frac{\mathrm{W}\left(1-\lambda_{c}\right)}{1+\beta}-\frac{1-\lambda_{c}}{1+\beta} \log \left(1-\lambda_{\mu}\right)+\frac{\lambda_{c}-\lambda_{\mu}}{1+\beta}\right. \\
& +\frac{C_{i} \alpha_{s}\left(\mu_{\mathrm{NP}}\right)}{\pi}\left[L_{g}^{2}-\frac{\left(L_{c}+(1+\beta) L_{g}-L_{\mu}\right)^{2}}{1+\beta}+2 L_{g}\left(L_{E}-L_{\mu}\right)\right] \text {, }
\end{aligned}
$$


with $\Delta_{E}^{(0)}=\left(z_{\text {cut }} \tilde{\mu}^{\beta}\right)^{1 /(1+\beta)}$. Finally, for $R_{g} / R_{0}<\left(\tilde{\mu} / z_{\text {cut }}\right)^{1 / \beta}$,

$$
\begin{aligned}
& \bar{R}_{2}\left(\Delta_{E}\right) \stackrel{\Delta_{E}>\Delta_{E}^{(0)}}{=} \quad \frac{C_{i}}{2 \pi \alpha_{s} \beta_{0}^{2}}\left[\frac{\mathrm{W}\left(1-\lambda_{c}\right)}{1+\beta}-\mathrm{W}\left(1-\lambda_{E}\right)+\frac{\beta}{1+\beta} \mathrm{W}\left(1+\frac{\lambda_{c}-(1+\beta) \lambda_{E}}{\beta}\right)\right] \\
& \tilde{\mu}<\Delta_{E}<\Delta_{E}^{(0)} \quad \frac{C_{i}}{2 \pi \alpha_{s} \beta_{0}^{2}}\left[\frac{\mathrm{W}\left(1-\lambda_{c}\right)}{1+\beta}-\mathrm{W}\left(1-\lambda_{E}\right)+\frac{\beta+\lambda_{c}-(1+\beta) \lambda_{E}}{1+\beta} \log \left(1-\lambda_{\mu}\right)\right. \\
& \left.+\frac{\lambda_{c}+\beta \lambda_{\mu}-(1+\beta) \lambda_{E}}{1+\beta}\right]+\frac{C_{i} \alpha_{s}\left(\mu_{\mathrm{NP}}\right)}{\pi} \frac{1+\beta}{\beta}\left(L_{E}-\frac{L_{c}+\beta L_{\mu}}{1+\beta}\right)^{2} \\
& z_{\text {cut }}\left(R_{g} / R_{0}\right)^{\beta}<\Delta_{E}<\tilde{\mu} \frac{C_{i}}{2 \pi \alpha_{s} \beta_{0}^{2}}\left[\frac{\mathrm{W}\left(1-\lambda_{c}\right)}{1+\beta}-\frac{1-\lambda_{c}}{1+\beta} \log \left(1-\lambda_{\mu}\right)+\frac{\lambda_{c}-\lambda_{\mu}}{1+\beta}\right] \\
& +\frac{C_{i} \alpha_{s}\left(\mu_{\mathrm{NP}}\right)}{\pi}\left[\frac{\left(L_{E}-L_{c}\right)^{2}}{\beta}-\frac{\left(L_{\mu}-L_{c}\right)^{2}}{1+\beta}\right] \\
& \Delta_{E}<z_{\mathrm{cut}}\left(R_{g} / R_{0}\right)^{\beta} \frac{C_{i}}{2 \pi \alpha_{s} \beta_{0}^{2}}\left[\frac{\mathrm{W}\left(1-\lambda_{c}\right)}{1+\beta}-\frac{1-\lambda_{c}}{1+\beta} \log \left(1-\lambda_{\mu}\right)+\frac{\lambda_{c}-\lambda_{\mu}}{1+\beta}\right] \\
& +\frac{C_{i} \alpha_{s}\left(\mu_{\mathrm{NP}}\right)}{\pi}\left[\beta L_{g}^{2}-\frac{\left(L_{\mu}-L_{c}\right)^{2}}{1+\beta}+2 L_{g}\left(L_{E}-L_{c}-\beta L_{g}\right)\right] .
\end{aligned}
$$

In the above expressions, we have introduced $L_{E}=\log \left(1 / \Delta_{E}\right)$ and $\lambda_{E}=2 \alpha_{s} \beta_{0} L_{E}$.

Open Access. This article is distributed under the terms of the Creative Commons Attribution License (CC-BY 4.0), which permits any use, distribution and reproduction in any medium, provided the original author(s) and source are credited.

\section{References}

[1] A. Abdesselam et al., Boosted objects: A Probe of beyond the Standard Model physics, Eur. Phys. J. C 71 (2011) 1661 [arXiv: 1012.5412] [INSPIRE].

[2] A. Altheimer et al., Jet Substructure at the Tevatron and LHC: New results, new tools, new benchmarks, J. Phys. G 39 (2012) 063001 [arXiv:1201.0008] [INSPIRE].

[3] A. Altheimer et al., Boosted objects and jet substructure at the LHC, arXiv:1311.2708 [INSPIRE].

[4] M.H. Seymour, Searches for new particles using cone and cluster jet algorithms: A Comparative study, Z.Phys. C62 (1994) 127.

[5] J.M. Butterworth, B.E. Cox and J.R. Forshaw, $W W$ scattering at the CERN LHC, Phys. Rev. D 65 (2002) 096014 [hep-ph/0201098] [INSPIRE].

[6] J.M. Butterworth, A.R. Davison, M. Rubin and G.P. Salam, Jet substructure as a new Higgs search channel at the LHC, Phys. Rev. Lett. 100 (2008) 242001 [arXiv:0802. 2470] [INSPIRE].

[7] L.G. Almeida et al., Substructure of high-p $p_{T}$ Jets at the LHC, Phys. Rev. D 79 (2009) 074017 [arXiv: 0807.0234] [INSPIRE].

[8] L.G. Almeida, S.J. Lee, G. Perez, I. Sung and J. Virzi, Top Jets at the LHC, Phys. Rev. D 79 (2009) 074012 [arXiv: 0810.0934] [INSPIRE]. 
[9] D.E. Kaplan, K. Rehermann, M.D. Schwartz and B. Tweedie, Top Tagging: A Method for Identifying Boosted Hadronically Decaying Top Quarks, Phys. Rev. Lett. 101 (2008) 142001 [arXiv:0806.0848] [INSPIRE].

[10] G. Brooijmans, High pt hadronic top quark identification, ATL-PHYS-CONF-2008-008 (2008).

[11] J. Thaler and L.-T. Wang, Strategies to Identify Boosted Tops, JHEP 07 (2008) 092 [arXiv: 0806.0023] [INSPIRE].

[12] CMS collaboration, A Cambridge-Aachen (C-A) based Jet Algorithm for boosted top-jet tagging, CMS-PAS-JME-09-001 (2009).

[13] CMS collaboration, Search for High Mass tt Resonances in the All-Hadronic Mode, CMS-PAS-EXO-09-002 (2009).

[14] S. Rappoccio, A new top jet tagging algorithm for highly boosted top jets, CMS-CR-2009-255 (2009).

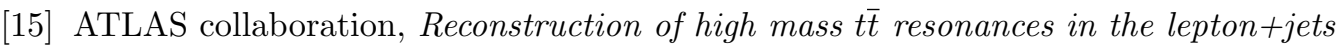
channel, ATL-PHYS-PUB-2009-081 (2009).

[16] G.D. Kribs, A. Martin, T.S. Roy and M. Spannowsky, Discovering the Higgs Boson in New Physics Events using Jet Substructure, Phys. Rev. D 81 (2010) 111501 [arXiv:0912.4731] [INSPIRE].

[17] G.D. Kribs, A. Martin, T.S. Roy and M. Spannowsky, Discovering Higgs Bosons of the MSSM using Jet Substructure, Phys. Rev. D 82 (2010) 095012 [arXiv:1006.1656] [INSPIRE].

[18] C.-R. Chen, M.M. Nojiri and W. Sreethawong, Search for the Elusive Higgs Boson Using Jet Structure at LHC, JHEP 11 (2010) 012 [arXiv:1006.1151] [INSPIRE].

[19] C. Hackstein and M. Spannowsky, Boosting Higgs discovery: The Forgotten channel, Phys. Rev. D 82 (2010) 113012 [arXiv: 1008.2202] [InSPIRE].

[20] A. Falkowski, D. Krohn, L.-T. Wang, J. Shelton and A. Thalapillil, Unburied Higgs boson: Jet substructure techniques for searching for Higgs' decay into gluons, Phys. Rev. D 84 (2011) 074022 [arXiv: 1006.1650] [INSPIRE].

[21] A. Katz, M. Son and B. Tweedie, Jet Substructure and the Search for Neutral Spin-One Resonances in Electroweak Boson Channels, JHEP 03 (2011) 011 [arXiv:1010.5253] [INSPIRE].

[22] Y. Cui, Z. Han and M.D. Schwartz, W-jet Tagging: Optimizing the Identification of Boosted Hadronically-Decaying W Bosons, Phys. Rev. D 83 (2011) 074023 [arXiv:1012.2077] [INSPIRE].

[23] J.-H. Kim, Rest Frame Subjet Algorithm With SISCone Jet For Fully Hadronic Decaying Higgs Search, Phys. Rev. D 83 (2011) 011502 [arXiv:1011.1493] [InSPIRE].

[24] J. Thaler and K. Van Tilburg, Identifying Boosted Objects with N-subjettiness, JHEP 03 (2011) 015 [arXiv: 1011.2268] [inSPIRE].

[25] J. Gallicchio et al., Multivariate discrimination and the Higgs $+W / Z$ search, JHEP 04 (2011) 069 [arXiv: 1010.3698] [inSPIRE].

[26] J. Gallicchio and M.D. Schwartz, Seeing in Color: Jet Superstructure, Phys. Rev. Lett. 105 (2010) 022001 [arXiv: 1001.5027] [InSPIRE]. 
[27] ATLAS collaboration, Prospects for top anti-top resonance searches using early atlas data, ATL-PHYS-PUB-2010-008.

[28] T. Plehn, G.P. Salam and M. Spannowsky, Fat Jets for a Light Higgs, Phys. Rev. Lett. 104 (2010) 111801 [arXiv:0910.5472] [INSPIRE].

[29] T. Plehn, M. Spannowsky, M. Takeuchi and D. Zerwas, Stop Reconstruction with Tagged Tops, JHEP 10 (2010) 078 [arXiv: 1006.2833] [inSPIRE].

[30] L.G. Almeida, S.J. Lee, G. Perez, G. Sterman and I. Sung, Template Overlap Method for Massive Jets, Phys. Rev. D 82 (2010) 054034 [arXiv:1006.2035] [InSPIRE].

[31] J. Thaler and K. Van Tilburg, Maximizing Boosted Top Identification by Minimizing N-subjettiness, JHEP 02 (2012) 093 [arXiv:1108.2701] [INSPIRE].

[32] M. Jankowiak and A.J. Larkoski, Jet Substructure Without Trees, JHEP 06 (2011) 057 [arXiv:1104.1646] [INSPIRE].

[33] A. Hook, M. Jankowiak and J.G. Wacker, Jet Dipolarity: Top Tagging with Color Flow, JHEP 04 (2012) 007 [arXiv:1102.1012] [InSPIRE].

[34] D.E. Soper and M. Spannowsky, Finding physics signals with shower deconstruction, Phys. Rev. D 84 (2011) 074002 [arXiv: 1102.3480] [InSPIRE].

[35] L.G. Almeida et al., Three-particle templates for a boosted Higgs boson, Phys. Rev. D 85 (2012) 114046 [arXiv:1112.1957] [INSPIRE].

[36] S.D. Ellis, A. Hornig, T.S. Roy, D. Krohn and M.D. Schwartz, Qjets: A Non-Deterministic Approach to Tree-Based Jet Substructure, Phys. Rev. Lett. 108 (2012) 182003 [arXiv: 1201.1914] [INSPIRE].

[37] D.E. Soper and M. Spannowsky, Finding top quarks with shower deconstruction, Phys. Rev. D 87 (2013) 054012 [arXiv:1211.3140] [INSPIRE].

[38] M. Backovic, J. Juknevich and G. Perez, Boosting the Standard Model Higgs Signal with the Template Overlap Method, JHEP 07 (2013) 114 [arXiv:1212.2977] [INSPIRE].

[39] T. Cohen, E. Izaguirre, M. Lisanti and H.K. Lou, Jet Substructure by Accident, JHEP 03 (2013) 161 [arXiv:1212.1456] [INSPIRE].

[40] D. Curtin, R. Essig and B. Shuve, Boosted Multijet Resonances and New Color-Flow Variables, Phys. Rev. D 88 (2013) 034019 [arXiv:1210.5523] [InSPIRE].

[41] S. El Hedri, A. Hook, M. Jankowiak and J.G. Wacker, Learning How to Count: A High Multiplicity Search for the LHC, JHEP 08 (2013) 136 [arXiv:1302.1870] [INSPIRE].

[42] M. Backovic, O. Gabizon, J. Juknevich, G. Perez and Y. Soreq, Measuring boosted tops in semi-leptonic t $\bar{t}$ events for the standard model and beyond, JHEP 04 (2014) 176 [arXiv:1311.2962] [INSPIRE].

[43] M. Gouzevitch et al., Scale-invariant resonance tagging in multijet events and new physics in Higgs pair production, JHEP 07 (2013) 148 [arXiv: 1303.6636] [INSPIRE].

[44] A.J. Larkoski, G.P. Salam and J. Thaler, Energy Correlation Functions for Jet Substructure, JHEP 06 (2013) 108 [arXiv:1305.0007] [INSPIRE].

[45] Y.-T. Chien, Telescoping Jets: Multiple Event Interpretations with Multiple R's, arXiv: 1304.5240 [INSPIRE]. 
[46] D. Kahawala, D. Krohn and M.D. Schwartz, Jet Sampling: Improving Event Reconstruction through Multiple Interpretations, JHEP 06 (2013) 006 [arXiv: 1304.2394] [INSPIRE].

[47] J. Gallicchio and M.D. Schwartz, Quark and Gluon Tagging at the LHC, Phys. Rev. Lett. 107 (2011) 172001 [arXiv: 1106.3076] [INSPIRE].

[48] J. Gallicchio and M.D. Schwartz, Quark and Gluon Jet Substructure, JHEP 04 (2013) 090 [arXiv:1211.7038] [INSPIRE].

[49] D. Krohn, M.D. Schwartz, T. Lin and W.J. Waalewijn, Jet Charge at the LHC, Phys. Rev. Lett. 110 (2013) 212001 [arXiv: 1209.2421] [INSPIRE].

[50] CMS collaboration, Search for a Higgs boson in the decay channel $H$ to $Z Z^{*} \rightarrow q \bar{q} \ell^{-} l^{+}$in pp collisions at $\sqrt{s}=7 \mathrm{TeV}$, JHEP 04 (2012) 036 [arXiv:1202.1416] [INSPIRE].

[51] F. Pandolfi and D. Del Re, Search for the Standard Model Higgs Boson in the $H \rightarrow Z Z \rightarrow$ llqq Decay Channel at CMS, PhD thesis, Zurich, ETH (2012).

[52] M. Cacciari and G.P. Salam, Pileup subtraction using jet areas, Phys. Lett. B 659 (2008) 119 [arXiv:0707.1378] [INSPIRE].

[53] D. Krohn, J. Thaler and L.-T. Wang, Jet Trimming, JHEP 02 (2010) 084 [arXiv: 0912.1342] [INSPIRE].

[54] S.D. Ellis, C.K. Vermilion and J.R. Walsh, Techniques for improved heavy particle searches with jet substructure, Phys. Rev. D 80 (2009) 051501 [arXiv:0903.5081] [INSPIRE].

[55] S.D. Ellis, C.K. Vermilion and J.R. Walsh, Recombination Algorithms and Jet Substructure: Pruning as a Tool for Heavy Particle Searches, Phys. Rev. D 81 (2010) 094023 [arXiv:0912.0033] [INSPIRE].

[56] R. Alon, E. Duchovni, G. Perez, A.P. Pranko and P.K. Sinervo, A Data-driven method of pile-up correction for the substructure of massive jets, Phys. Rev. D 84 (2011) 114025 [arXiv:1101.3002] [INSPIRE].

[57] G. Soyez, G.P. Salam, J. Kim, S. Dutta and M. Cacciari, Pileup subtraction for jet shapes, Phys. Rev. Lett. 110 (2013) 162001 [arXiv:1211.2811] [InSPIRE].

[58] J. Tseng and H. Evans, Sequential recombination algorithm for jet clustering and background subtraction, Phys. Rev. D 88 (2013) 014044 [arXiv: 1304.1025] [INSPIRE].

[59] M. Dasgupta, A. Fregoso, S. Marzani and G.P. Salam, Towards an understanding of jet substructure, JHEP 09 (2013) 029 [arXiv:1307.0007] [INSPIRE].

[60] M. Dasgupta, A. Fregoso, S. Marzani and A. Powling, Jet substructure with analytical methods, Eur. Phys. J. C 73 (2013) 2623 [arXiv:1307.0013] [INSPIRE].

[61] D. Krohn, M.D. Schwartz, M. Low and L.-T. Wang, Jet Cleansing: Pileup Removal at High Luminosity, arXiv:1309.4777 [INSPIRE].

[62] ATLAS collaboration, Jet substructure in ATLAS, arXiv:1110.1094 [ATL-PHYS-PROC-2011-142] [INSPIRE].

[63] ATLAS collaboration, Search for resonances decaying into top-quark pairs using fully hadronic decays in pp collisions with ATLAS at $\sqrt{s}=7$ TeV, JHEP 01 (2013) 116 [arXiv: 1211.2202] [INSPIRE].

[64] ATLAS collaboration, Search for pair production of massive particles decaying into three quarks with the ATLAS detector in $\sqrt{s}=7 \mathrm{TeV}$ pp collisions at the LHC, JHEP 12 (2012) 086 [arXiv: 1210.4813] [INSPIRE]. 
[65] ATLAS collaboration, ATLAS measurements of the properties of jets for boosted particle searches, Phys. Rev. D 86 (2012) 072006 [arXiv: 1206.5369] [INSPIRE].

[66] ATLAS collaboration, Measurement of jet mass and substructure for inclusive jets in $\sqrt{s}=$ 7 tev pp collisions with the atlas experiment, ATLAS-CONF-2011-073 (2011).

[67] ATLAS collaboration, Light-quark and gluon jets in ATLAS, ATLAS-CONF-2011-053 (2011).

[68] ATLAS collaboration, Jet mass and substructure of inclusive jets in $\sqrt{s}=7 \mathrm{TeV} \mathrm{pp}$ collisions with the ATLAS experiment, JHEP 05 (2012) 128 [arXiv:1203.4606] [INSPIRE].

[69] ATLAS collaboration, Studies of the impact and mitigation of pile-up on large- $R$ and groomed jets in ATLAS at $\sqrt{s}=7 \mathrm{TeV}$, ATLAS-CONF-2012-066 (2012).

[70] ATLAS collaboration, Performance of large-r jets and jet substructure reconstruction with the atlas detector, ATLAS-CONF-2012-065 (2012).

[71] ATLAS collaboration, Studies of the impact and mitigation of pile-up on large-r and groomed jets in atlas at $\sqrt{s}=7$ tev, ATLAS-CONF-2012-066 (2012).

[72] ATLAS collaboration, Measurement of jet shapes in top-quark pair events at $\sqrt{s}=7 \mathrm{TeV}$ using the ATLAS detector, Eur. Phys. J. C 73 (2013) 2676 [arXiv:1307.5749] [InSPIRE].

[73] ATLAS collaboration, Performance of jet substructure techniques for large- $R$ jets in proton-proton collisions at $\sqrt{s}=7 \mathrm{TeV}$ using the ATLAS detector, JHEP 09 (2013) 076 [arXiv: 1306.4945] [INSPIRE].

[74] ATLAS collaboration, Pile-up subtraction and suppression for jets in ATLAS, ATLAS-CONF-2013-083 (2013).

[75] ATLAS collaboration, Performance of boosted top quark identification in 2012 ATLAS data, ATLAS-CONF-2013-084 (2013).

[76] ATLAS collaboration, Performance of pile-up subtraction for jet shapes, ATLAS-CONF-2013-085 (2013).

[77] ATLAS collaboration, Jet Charge Studies with the ATLAS Detector Using $\sqrt{s}=8 \mathrm{TeV}$ Proton-Proton Collision Data, ATLAS-CONF-2013-086 (2013).

[78] ATLAS collaboration, Performance and Validation of Q-Jets at the ATLAS Detector in pp Collisions at $\sqrt{s}=8 \mathrm{TeV}$ in 2012, ATLAS-CONF-2013-087 (2013).

[79] ATLAS collaboration, Identification and Tagging of Double b-hadron jets with the ATLAS Detector, ATLAS-CONF-2012-100 (2012).

[80] CMS collaboration, Jet substructure algorithms, CMS-PAS-JME-10-013 (2011).

[81] CMS collaboration, Search for BSM ttbar Production in the Boosted All-Hadronic Final State, CMS-PAS-EXO-11-006 (2011).

[82] CMS collaboration, Measurement of the subjet multiplicity in dijet events from proton-proton collisions at $\sqrt{s}=7 \mathrm{TeV}$, CMS-PAS-QCD-10-041 (2010).

[83] CMS collaboration, Measurement of the underlying event activity in pp collisions at $\sqrt{s}=0.9$ and $7 \mathrm{TeV}$ with the novel jet-area/median approach, JHEP 08 (2012) 130 [arXiv: 1207.2392] [INSPIRE].

[84] CMS collaboration, Performance of quark/gluon discrimination in $8 \mathrm{TeV}$ pp data, CMS-PAS-JME-13-002 (2013). 
[85] CMS collaboration, Pileup Jet Identification, CMS-PAS-JME-13-005 (2013).

[86] CMS collaboration, Performance of b tagging at $\sqrt{s}=8 \mathrm{Te} V$ in multijet, ttbar and boosted topology events, CMS-PAS-BTV-13-001 (2013).

[87] CMS collaboration, Identifying Hadronically Decaying Vector Bosons Merged into a Single Jet, CMS-PAS-JME-13-006 (2013).

[88] CMS collaboration, Shape, transverse size and charged hadron multiplicity of jets in pp collisions at 7 TeV, JHEP 06 (2012) 160 [arXiv:1204.3170] [INSPIRE].

[89] CMS collaboration, Studies of jet mass in dijet and $W / Z+$ jet events, JHEP 05 (2013) 090 [arXiv: 1303.4811] [InSPIRE].

[90] H.-n. Li, Z. Li and C.-P. Yuan, QCD resummation for light-particle jets, Phys. Rev. D 87 (2013) 074025 [arXiv: 1206.1344] [INSPIRE].

[91] S.D. Ellis, C.K. Vermilion, J.R. Walsh, A. Hornig and C. Lee, Jet Shapes and Jet Algorithms in SCET, JHEP 11 (2010) 101 [arXiv:1001.0014] [INSPIRE].

[92] A. Banfi, M. Dasgupta, K. Khelifa-Kerfa and S. Marzani, Non-global logarithms and jet algorithms in high-pT jet shapes, JHEP 08 (2010) 064 [arXiv:1004.3483] [INSPIRE].

[93] M. Dasgupta, K. Khelifa-Kerfa, S. Marzani and M. Spannowsky, On jet mass distributions in $Z+$ jet and dijet processes at the LHC, JHEP 10 (2012) 126 [arXiv:1207.1640] [INSPIRE].

[94] Y.-T. Chien, R. Kelley, M.D. Schwartz and H.X. Zhu, Resummation of Jet Mass at Hadron Colliders, Phys. Rev. D 87 (2013) 014010 [arXiv:1208.0010] [InSPIRE].

[95] T.T. Jouttenus, I.W. Stewart, F.J. Tackmann and W.J. Waalewijn, Jet mass spectra in Higgs boson plus one jet at next-to-next-to-leading logarithmic order, Phys. Rev. D 88 (2013) 054031 [arXiv: 1302.0846] [INSPIRE].

[96] M. Rubin, Non-Global Logarithms in Filtered Jet Algorithms, JHEP 05 (2010) 005 [arXiv:1002 .4557] [INSPIRE].

[97] J.R. Walsh and S. Zuberi, Factorization Constraints on Jet Substructure, arXiv:1110.5333 [INSPIRE].

[98] I. Feige, M.D. Schwartz, I.W. Stewart and J. Thaler, Precision Jet Substructure from Boosted Event Shapes, Phys. Rev. Lett. 109 (2012) 092001 [arXiv:1204.3898] [INSPIRE].

[99] M. Field, G. Gur-Ari, D.A. Kosower, L. Mannelli and G. Perez, Three-Prong Distribution of Massive Narrow QCD Jets, Phys. Rev. D 87 (2013) 094013 [arXiv:1212.2106] [INSPIRE].

[100] A.J. Larkoski, QCD Analysis of the Scale-Invariance of Jets, Phys. Rev. D 86 (2012) 054004 [arXiv: 1207. 1437] [INSPIRE].

[101] E. Gerwick, S. Schumann, B. Gripaios and B. Webber, QCD Jet Rates with the Inclusive Generalized kt Algorithms, JHEP 04 (2013) 089 [arXiv: 1212.5235] [INSPIRE].

[102] A.J. Larkoski, D. Neill and J. Thaler, Jet Shapes with the Broadening Axis, JHEP 04 (2014) 017 [arXiv: 1401.2158] [INSPIRE].

[103] A.J. Larkoski, I. Moult and D. Neill, Toward Multi-Differential Cross Sections: Measuring Two Angularities on a Single Jet, arXiv:1401.4458 [INSPIRE].

[104] M. Dasgupta and G.P. Salam, Resummation of nonglobal QCD observables, Phys. Lett. B 512 (2001) 323 [hep-ph/0104277] [INSPIRE]. 
[105] A.J. Larkoski and J. Thaler, Unsafe but Calculable: Ratios of Angularities in Perturbative QCD, JHEP 09 (2013) 137 [arXiv: 1307.1699] [InSPIRE].

[106] A. Banfi, G.P. Salam and G. Zanderighi, Principles of general final-state resummation and automated implementation, JHEP 03 (2005) 073 [hep-ph/0407286] [INSPIRE].

[107] C.F. Berger, T. Kucs and G.F. Sterman, Event shape/energy flow correlations, Phys. Rev. D 68 (2003) 014012 [hep-ph/0303051] [INSPIRE].

[108] M. Cacciari, G.P. Salam and G. Soyez, The Catchment Area of Jets, JHEP 04 (2008) 005 [arXiv:0802.1188] [INSPIRE].

[109] M. Cacciari, G.P. Salam and G. Soyez, The Anti-k(t) jet clustering algorithm, JHEP 04 (2008) 063 [arXiv:0802.1189] [InSPIRE].

[110] Y.L. Dokshitzer, G.D. Leder, S. Moretti and B.R. Webber, Better jet clustering algorithms, JHEP 08 (1997) 001 [hep-ph/9707323] [INSPIRE].

[111] M. Wobisch and T. Wengler, Hadronization corrections to jet cross-sections in deep inelastic scattering, hep-ph/9907280 [INSPIRE].

[112] S. Catani, Y.L. Dokshitzer, M.H. Seymour and B.R. Webber, Longitudinally invariant $K_{t}$ clustering algorithms for hadron hadron collisions, Nucl. Phys. B 406 (1993) 187 [INSPIRE].

[113] S.D. Ellis and D.E. Soper, Successive combination jet algorithm for hadron collisions, Phys. Rev. D 48 (1993) 3160 [hep-ph/9305266] [INSPIRE].

[114] S. Catani, L. Trentadue, G. Turnock and B.R. Webber, Resummation of large logarithms in $e^{+} e^{-}$event shape distributions, Nucl. Phys. B 407 (1993) 3 [INSPIRE].

[115] Y.L. Dokshitzer, A. Lucenti, G. Marchesini and G.P. Salam, On the QCD analysis of jet broadening, JHEP 01 (1998) 011 [hep-ph/9801324] [INSPIRE].

[116] A. Banfi, G.P. Salam and G. Zanderighi, Principles of general final-state resummation and automated implementation, JHEP 03 (2005) 073 [hep-ph/0407286] [INSPIRE].

[117] Y. Hatta and T. Ueda, Resummation of non-global logarithms at finite $N_{c}$, Nucl. Phys. B 874 (2013) 808 [arXiv:1304.6930] [INSPIRE].

[118] A. Banfi and M. Dasgupta, Problems in resumming interjet energy flows with $k_{t}$ clustering, Phys. Lett. B 628 (2005) 49 [hep-ph/0508159] [INSPIRE].

[119] Y.L. Dokshitzer, G. Marchesini and G. Oriani, Measuring color flows in hard processes: Beyond leading order, Nucl. Phys. B 387 (1992) 675 [INSPIRE].

[120] T. Sjöstrand, S. Mrenna and P.Z. Skands, A Brief Introduction to PYTHIA 8.1, Comput. Phys. Commun. 178 (2008) 852 [arXiv:0710.3820] [INSPIRE].

[121] R. Corke and T. Sjöstrand, Interleaved Parton Showers and Tuning Prospects, JHEP 03 (2011) 032 [arXiv:1011.1759] [INSPIRE].

[122] M. Dasgupta, L. Magnea and G.P. Salam, Non-perturbative QCD effects in jets at hadron colliders, JHEP 02 (2008) 055 [arXiv:0712.3014] [INSPIRE].

[123] M. Cacciari, G.P. Salam and G. Soyez, FastJet User Manual, Eur. Phys. J. C 72 (2012) 1896 [arXiv: 1111.6097] [INSPIRE].

[124] M. Cacciari, G.P. Salam and G. Soyez, The Catchment Area of Jets, JHEP 04 (2008) 005 [arXiv: 0802.1188] [INSPIRE]. 
[125] S. Sapeta, Q.C. Zhang and Q.C. Zhang, The mass area of jets, JHEP 06 (2011) 038 [arXiv: 1009.1143] [INSPIRE].

[126] S. Catani, B.R. Webber and G. Marchesini, QCD coherent branching and semiinclusive processes at large x, Nucl. Phys. B 349 (1991) 635 [InSPIRE].

[127] D. Bertolini, T. Chan and J. Thaler, Jet Observables Without Jet Algorithms, JHEP 04 (2014) 013 [arXiv: 1310.7584] [INSPIRE].

[128] R. Abbate, M. Fickinger, A.H. Hoang, V. Mateu and I.W. Stewart, Thrust at $N^{3} L L$ with Power Corrections and a Precision Global Fit for $\alpha s_{m Z}$, Phys. Rev. D 83 (2011) 074021 [arXiv: 1006.3080] [INSPIRE]. 